\title{
The onset of p53 loss of heterozygosity is differentially induced in various stem cell types and may involve the loss of either allele
}

\author{
Y Shetzer ${ }^{1,4}$, S Kagan ${ }^{1,4}$, G Koifman ${ }^{1}$, R Sarig', I Kogan-Sakin ${ }^{1}$, M Charni ${ }^{1}$, T Kaufman ${ }^{1}$, M Zapatka ${ }^{2}$, A Molchadsky ${ }^{1}$,

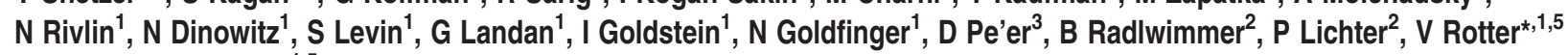 \\ and R Aloni-Grinstein ${ }^{1,5}$
}

p53 loss of heterozygosity (p53LOH) is frequently observed in Li-Fraumeni syndrome (LFS) patients who carry a mutant (Mut) p53 germ-line mutation. Here, we focused on elucidating the link between p53LOH and tumor development in stem cells (SCs). Although adult mesenchymal stem cells (MSCs) robustly underwent p53LOH, p53LOH in induced embryonic pluripotent stem cells (iPSCs) was significantly attenuated. Only SCs that underwent p53LOH induced malignant tumors in mice. These results may explain why LFS patients develop normally, yet acquire tumors in adulthood. Surprisingly, an analysis of single-cell sub-clones of iPSCs, MSCs and ex vivo bone marrow (BM) progenitors revealed that p53LOH is a bi-directional process, which may result in either the loss of wild-type (WT) or Mut p53 allele. Interestingly, most BM progenitors underwent Mutp53LOH. Our results suggest that the bi-directional p53LOH process may function as a cell-fate checkpoint. The loss of Mutp53 may be regarded as a DNA repair event leading to genome stability. Indeed, gene expression analysis of the p53LOH process revealed upregulation of a specific chromatin remodeler and a burst of DNA repair genes. However, in the case of loss of WTp53, cells are endowed with uncontrolled growth that promotes cancer.

Cell Death and Differentiation (2014) 21, 1419-1431; doi:10.1038/cdd.2014.57; published online 16 May 2014

Heterozygosity, caused by a mutation in a single allele of a tumor suppressor gene (TSG), is one of the first steps in malignant transformation. ${ }^{1}$ Often, TSGs undergo loss of the wild-type (WT) allele, designated as loss of heterozygosity $(\mathrm{LOH}){ }^{2-4}$ Patients with the rare cancer predisposition Li-Fraumeni syndrome (LFS), carrying germ-line heterozygous p53 mutations, ${ }^{5}$ apparently exhibit normal development yet later in adult life develop a wide spectrum of tumors; predominantly sarcomas, ${ }^{6-8}$ where $40-60 \%$ of tumors exhibit WT p53 loss of heterozygosity (p53LOH). ${ }^{8}$

Giving that cancer development could be associated with stemness deregulation challenges, the notion that the occurrence of p53LOH in stem cells (SCs) may contribute to the emergence of cancer SCs. Genomic fidelity is a hallmark of SCs. ${ }^{9}$ The genome of embryonic stem cells (ESCs) is extremely stable, whereas adult stem cells (ASCs) exhibit a less stable genome. ${ }^{10}$ Genetic deregulation in ASCs was shown to be associated with tumor development. ${ }^{11-13}$ Mesenchymal stem cells (MSCs) that acquire mutations in oncogenes/TSGs such as p53 may function as tumor-initiating cells leading to de-novo sarcomagensis. ${ }^{14-17}$ Furthermore, MSCs isolated from young mice, aged in culture acquired clinically relevant p53 mutations. ${ }^{18}$ In all, these findings suggest a link between p53 inactivation in SCs and tumorigenesis.

Although induced pluripotent stem cells (iPSCs) seemed to represent ESCs, ${ }^{19,20}$ several studies questioned the assumption that iPSCs are as genomically stable as ESCs. ${ }^{21-24}$ p53 was found to have a major role in the generation of iPSCs both in attenuating reprogramming and controlling the quality of the reprogrammed cells. ${ }^{25,26}$ An additional role of p53 during reprogramming may be an indirect effect on cell proliferation ${ }^{27}$ and on the restriction of mesenchymal-epithelial transition during the early phases of reprogramming. ${ }^{28}$ Importantly, Mutp53 cells exhibiting a fully reprogrammed iPSC phenotype in vitro, form malignant tumors in vivo, instead of the benign teratomas induced by the WTp53-iPSCs. ${ }^{25}$ As p53 is the guardian of the genome, it is important to investigate how p53LOH would affect genome stability and tumorigenicity of iPSCs.

\footnotetext{
${ }^{1}$ Department of Molecular Cell Biology, Weizmann Institute of Science, Rehovot, Israel; ${ }^{2}$ Division of Molecular Genetics, German Cancer Research Center (DKFZ), Im Neuenheimer Feld 280, Heidelberg, Germany and ${ }^{3}$ Department of Biological Sciences, Columbia University, New York, NY, USA

${ }^{*}$ Corresponding author: V Rotter, Department of Molecular Cell Biology, Weizmann Institute of Science, Rehovot 76100, Israel. Tel: +972 89344501 ; Fax: +972 8 9465265; E-mail: varda.rotter@weizmann.ac.il

${ }^{4}$ These authors contributed equally to this study.

${ }^{5}$ These authors share equal senior authorship.

Abbreviations: LOH, loss of heterozygosity; LFS, Li-Fraumeni syndrome; SCs, stem cells; iPSCs, induced pluripotent stem cells; MSCs, mesenchymal stem cells; BM, bone marrow; WT, wild type; Mut, mutant; TSG, tumor suppressor gene; ESCs, embryonic stem cells; ASCs, adult stem cells; HZ, heterozygous; MEFs, mouse embryonic fibroblasts; SKY, spectral karyotyping; SNV, single-nucleotide variant; HRDRP, homologous recombination (HR) DNA repair pathway; NHEJ, non-homologous end joining; CFU-Fs, colony-forming units-fibroblast; p53LOH, p53 loss of heterozygosity; H\&E, hematoxylin and eosin; QRT-PCR, quantitative real-time PCR

Received 21.10.13; revised 27.2.14; accepted 17.3.14; Edited by B Zhivotovsky; published online 16.5.14
} 
The availability of in vitro SC p53LOH models (iPSCs, MSCs) can help decipher the role of p53LOH in cancer initiation. Indeed, the incidence of p53LOH was found to be extremely different between these SCs. Surprisingly, we found that reprograming of heterozygous p53 (HZp53) fibroblasts, which frequently undergo $\mathrm{p} 53 \mathrm{LOH}$, gave rise to iPSC clones, most of which retained their HZp53 status and exhibited features of normal WTp53-iPSCs. However, p53LOH process is robust in MSCs. Interestingly, single-cell sub-cloning of iPSCs, MSCs and ex vivo bone marrow (BM) progenitors revealed that, in addition to the loss of the WTp53, loss of the Mutp53 allele also takes place. Of note, this bi-directional p53LOH occurred in an age-dependent manner linking $\mathrm{LOH}$ to aging and tumorigenesis. Surprisingly, most of the p53LOH events in BM progenitors preferred the loss of the Mutp53 allele. Taken together, our results of a bi-directional p53LOH process, accompanied by a burst of DNA repair pathways, may suggest that $\mathrm{p} 53 \mathrm{LOH}$ can be regarded as a DNA repair event. In the case of a DNA repair-orientated productive LOH process, where the Mutp53 allele is lost, cells are rescued of tumorigenesis. However, when the WTp53 allele is lost, cells become prone to tumor initiation.

\section{Results}

Mouse embryonic fibroblasts (MEFs) undergo p53LOH in vitro. p53LOH may lead to cancer, yet little is known about this process in SCs. Our study focused on SCs (iPSCs and MSCs) generated or derived from mice heterozygous for the p53R172H mutation (Supplementary Figure 1). ${ }^{6}$ iPSCs are generated from MEFs, therefore we first examined whether HZp53-MEFs undergo p53LOH in vitro and found that WTp53-LOH occurred in $100 \%$ of examined MEFs at day 12 (passage 7). This correlated with a distinct shift in their proliferation capacity (Figures $1 a$ and $b$ ) and with the decrease of p21 mRNA and protein levels (Figures 1c and d), indicating loss of WTp53 function. Our results suggest that in
MEFs with one copy of WTp53 exhibited controlled cell growth, yet Mutp53 facilitates cell proliferation only upon the completion of WTp53-LOH.

Reprogramming attenuates LOH in HZp53-iPSCs. p53 status affects the oncogenic potential of iPSCs. ${ }^{25}$ Nevertheless, the oncogenic potential of HZp53-iPSCs is still unknown. To that end, WTp53, HZp53 and Mutp53-MEFs were reprogrammed into iPSCs. Interestingly, the early reprogramming kinetics of the HZp53 cells were comparable to those of WTp53 cells, as shown by the early reprogramming marker alkaline phosphatase (Figure 2a) and the rate of appearance of Nanog-expressing colonies (Figure 2b). The p53 status did not influence proliferation or apoptosis (Supplementary Figures $2 \mathrm{~A}$ and B). Suggesting that in the early steps of reprogramming of HZp53-iPSCs, the WTp53 dominates over the Mutp53. In contrast to MEFs, only $23 \%$ of HZp53-iPSC clones (6/26) underwent p53LOH (Figure 2c, Supplementary Table 1). This suggests that the reprogramming process attenuated p53LOH in iPSCs.

To study the effect of p53LOH on the genomic stability of the generated iPSC clones, we performed spectral karyotyping (SKY). ${ }^{29}$ iPSCs that did not undergo p53LOH largely exhibited a normal karyotype (Supplementary Figure $3 \mathrm{~A}$ ), whereas those that underwent p53LOH showed translocations and irregular chromosome numbers (Supplementary Figure 3B). Interestingly, we observed that HZp53 ESCs did not undergo p53LOH under prolonged culturing (submitted Rivlin et al.), suggesting that p53LOH is a rare event in genomically stable SCs.

To unravel the mechanism underlying $\mathrm{LOH}$, we examined the genotypic status by a Taqman quantitative real-time PCR (QRT-PCR) copy number assay (Figure 2d) and by sequencing. Interestingly, clones that lost the WTp53 allele duplicated the Mut allele and became homozygous rather than hemizygous (null/Mut), while the majority of clones retained their heterozygosity (Figure 2e). Furthermore, MEFs,
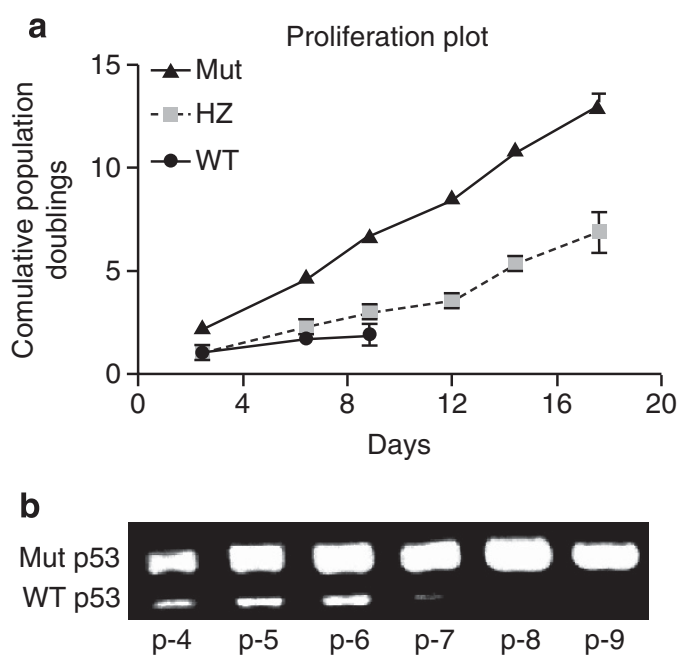
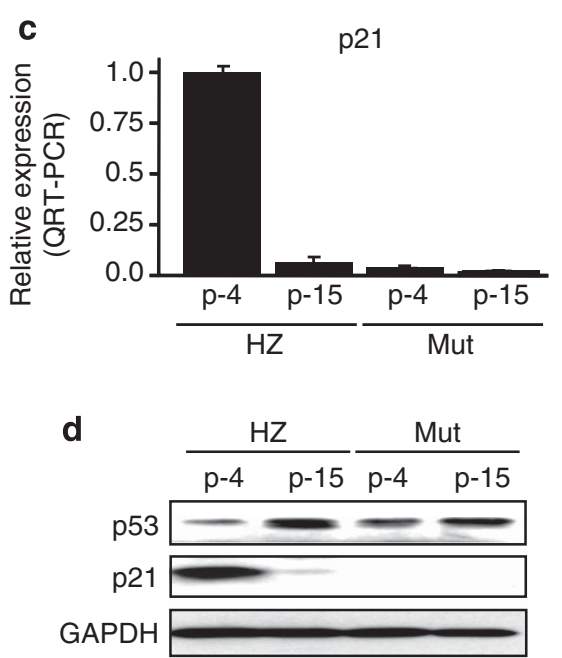

Figure 1 MEFs undergo p53LOH. MEFs derived from mice heterozygous for the murine R172H hot spot p53 mutation (HZp53) analogous to the human p53R175H hot spot mutation, as well as MEFs obtained from the corresponding WTp53 and mutant p53 (Mutp53) controls, were cultured and propagated in vitro. (a) Cumulative population doublings of WTp53, HZp53 and Mutp53-MEFs were calculated and plotted. (b) Genotyping analysis of p53 at various passages. (c) Relative mRNA expression of p21 in $\mathrm{HZ}$ and Mutp53-MEFs at early and late passages as measured by QRT-PCR. (d) Western blot analysis of p21 protein levels 
which were heterozygous at passage 3 , underwent duplication of the Mut allele at passage 9 (Figure 2f), as in iPSCs. These findings suggest that mitotic recombination or nondisjunction with duplication is the molecular mechanism involved in the $\mathrm{LOH}$ process. Whole-exome sequencing of four HZp53-iPSC clones and two HZp53-MEFs batches confirmed that three of the iPSCs clones retained their p53 heterozygosity, whereas one underwent p53LOH and all
MEFs displayed a p53LOH pattern. Interestingly, a singlenucleotide variant (SNV) in Efnb3, an adjacent gene upstream of Trp53 remained heterozygous in all examined samples regardless whether p53LOH occurred or not (Figure $2 \mathrm{~g}$ ). We concluded that homologous recombination $(\mathrm{HR})$ was the mechanism involved in the p53LOH process. Moreover, it is intriguing to speculate that a fragile site lies in between Efnb3 and Trp53. To examine whether MEF cultures contain a small

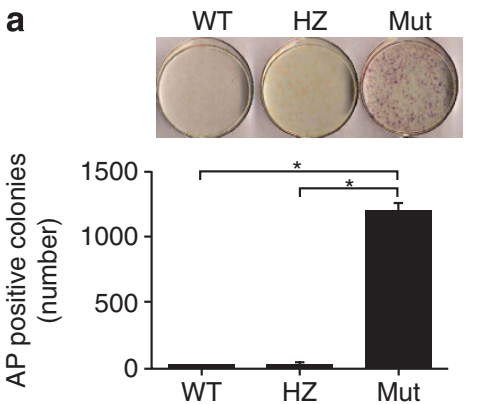

b
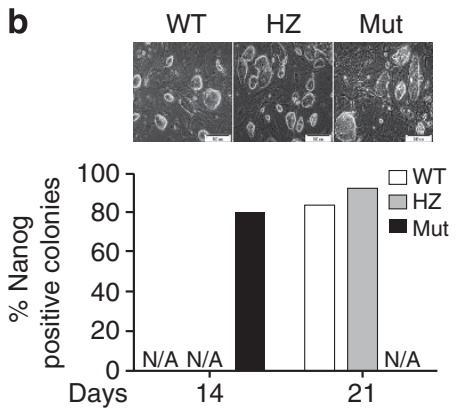

C
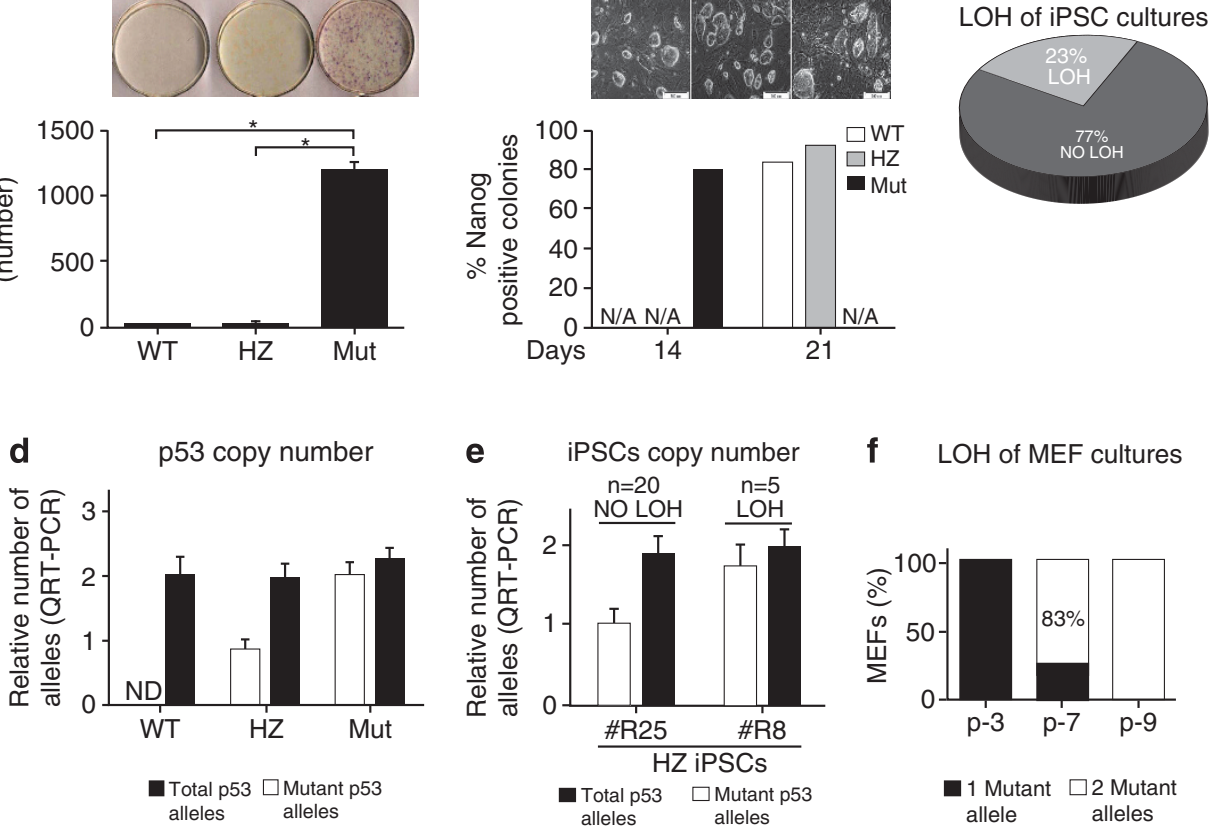

f LOH of MEF cultures

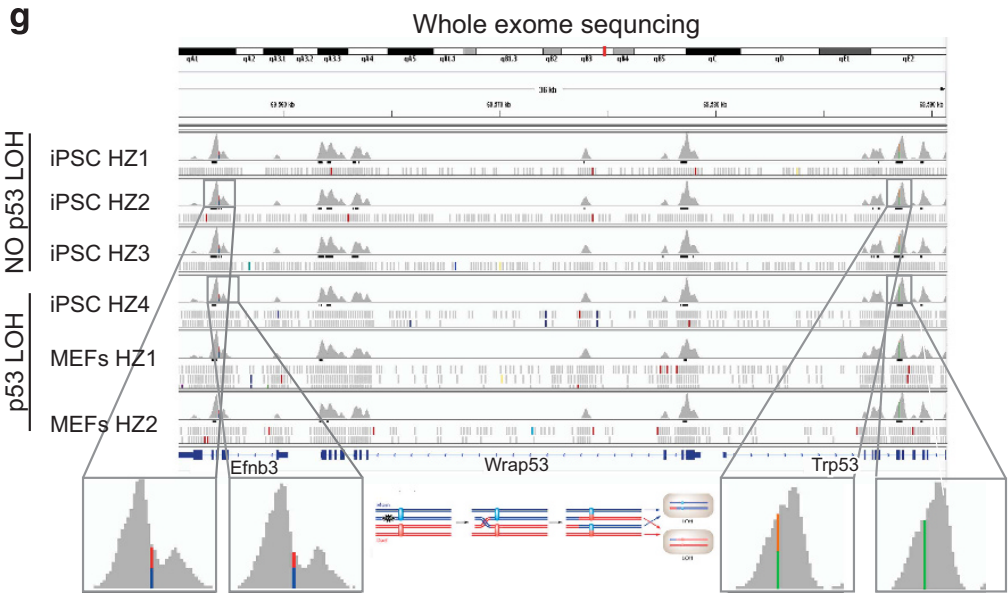

Figure 2 Reprogramming kinetics of HZp53-MEFs. (a) WTp53, HZp53 and Mutp53-MEFs were infected with lentiviruses encoding Oct4, Sox2 and Klf4. Cells were plated and assayed for alkaline phosphatase (AP) activity, 2 weeks post infection. A representative image (top) of the plates and quantification (bottom) are presented. ${ }^{*} P=0.0001$ one-way analysis of variance. (b) Light microscopy images obtained from the corresponding iPSC clones, depicting typical ES morphology. Lower panel: percentage of Nanog-positive colonies of WTp53, HZp53 and Mutp53 as measured by QRT-PCR, 14 and 21 days post infection. Nanog positivity was determined when Nanog mRNA expression was at least 50\% that of ES cells. (c) p53 PCR genotype and sequencing of $26 \mathrm{HZp53-iPSC}$ clones followed until p-40. Summary of the data from three independent experiments is presented in a pie chart. (d) Number of genomic p53 DNA copies was measured by Taqman QRT-PCR in WTp53, HZp53 and Mutp53-MEFs. Each well is normalized to the TFRC control gene. Total p53 and Mutp53 probes are designed to exon1 and loxP site, respectively. (e) A representative Taqman QRT-PCR of p53 copy number in HZ p53 iPSCs clones at p-12, 70 days post infection (\#R25 and \#R8). Probes and normalization were as in d. $n=25$. (f) A plot summarizing copy number of the mutant alleles of HZp53-MEFs in culture. (g) Four HZp53-iPSC clones at p-11 and two HZp53 MEF preparations at p-10 were subjected to whole-exome sequencing. Integrative Genomic Viewer image of a 36-kb section of chromosome 11 of the six samples is presented. Insets depict heterozygous SNVs in Efnb3 found in iPSC $\mathrm{HZ2}$ and HZ4, whereas these same cells are either heterozygous at a SNV in Trp53 (iPSC HZ2, two colors) or homozygous (iPSC HZ4, single color). At the bottom is shown a schematic representation of $\mathrm{p} 53 \mathrm{LOH}$ through homologous recombination 
sub-population of cells that retain their heterozygosity in culture, we re-examined the sequencing data of the two MEF samples. Indeed, between 1 and $11 \%$ of the reads were of the WTp53 allele. Hence, between 2 and $22 \%$ of the cells remained heterozygous for $\mathrm{p} 53$. One may speculate that the reprogramming itself is biased toward these cells, accounting for the low rates of p53LOH in iPSCs. However, the fact that homozygous Mutp53 facilitates reprogramming ${ }^{25}$ (Figures 2a and b), argues against this explanation. Hypothetically, chromosomal mis-pairing, as a result of the LoxP insertion, could cause recombination-related effects. To address this point, analysis of minor allele frequency in the examined genomes revealed a few $\mathrm{LOH}$ events in distinct regions in similar frequencies as chromosome 11 that entails $\operatorname{Trp} 53^{30}$ (Supplementary Figure 4). Hence, p53LOH is not due to the LoxP site. Altogether, reprogramming attenuates p53LOH, which occurs by duplication of the mutation via HR.
LOH in HZp53-iPSCs leads to malignancy. Despite the low frequency of $\mathrm{p} 53 \mathrm{LOH}$, it is important to evaluate the outcome of p53LOH on the malignant capacity of HZp53iPSCs. Injection of HZp53-iPSCs induced typical teratomas with well-differentiated regions of all three germ layers (Figure 3a), similar to that observed with the WTp53-iPSCs. However, injection of iPSC clones that underwent WTp53$\mathrm{LOH}$ in vitro (before their injection) induced malignant tumors (Figure 3c), like those induced by Mutp53-iPSCs (Figure 3d). This reiterates the idea that WTp53 is essential for proper differentiation of iPSCs and that loss of WTp53, giving rise to iPSCs exclusively expressing Mutp53, leads to the development of malignant tumors.

Interestingly, some of the short-term in vitro cultivated HZp53-iPSCs, initially characterized as heterozygous cells, generated tumors when injected into mice, exhibiting heterogeneous phenotypes of fully malignant tumor cells with

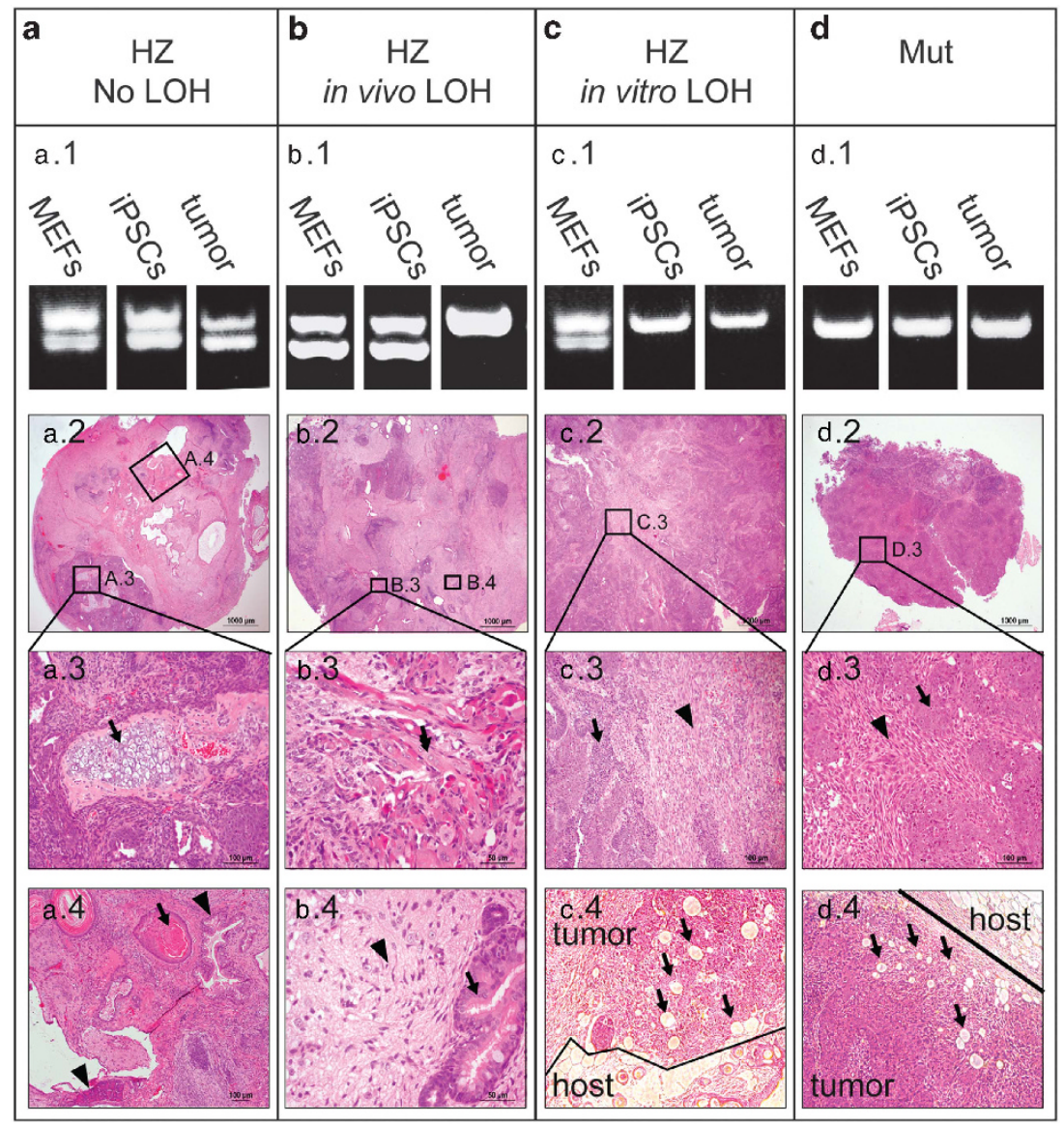

Figure 3 Histological sections of HZp53-iPSC-derived tumors. WTp53 and HZp53 that did not undergo p53LOH, HZp53 that underwent p53LOH in vivo, HZp53 that underwent p53LOH in vitro and Mutp53 iPSC clones were injected sub-cutaneously into nude mice. (a) Representative sections of no-LOH HZp53 clones, $n=10$. (a.1) PCR analysis of the original MEFs, injected iPSCs and the removed tumor. (a.2) A highly differentiated teratoma. (a.3) Arrow indicating cartilage (mesoderm). (a.4) Arrow indicating keratin (ectoderm) and an upper arrowhead indicating respiratory epithelium (endoderm). The lower arrowhead identifies exocrine pancreatic glands (endoderm). (b) Representative sections of HZp53 clones that underwent $\mathrm{LOH}$ in vivo, $n=4$. (b.1) As in a.1. (b.2) Predominantly well-differentiated teratoma. (b.3) Arrow indicating skeletal muscle fibers (mesoderm). (b.4) Arrow indicating respiratory epithelium (endoderm) and an arrowhead indicating well-differentiated nervous tissue (ectoderm) (c) Representative sections of HZp53 clones that underwent LOH in vitro, $n=5$. (c.1) As in a.1. (c.2) Mostly poorly differentiated tumor. (c.3) Arrow indicating nervous tissue (ectoderm) and an arrowhead indicating poorly differentiated stroma (mesenchyme), with features of sarcoma. (c.4) Tumor with invasive fronts. Line indicates the border between host tissue and the tumor. Arrows indicate host adipocytes engrafted by the tumor. (d) Representative sections of Mutp53 clones, $n=8$. (d.1) As in a.1. (d.2) A poorly differentiated tumor. (d.3) Arrow indicating nervous tissue (ectoderm) and an arrowhead indicating poorly differentiated stroma (mesenchyme), with features of sarcoma. (d.4) As in c.4. All sections were stained by H\&E 
an occasional incidence of differentiated teratoma (Figure 3b). This observation may suggest that these HZp53-iPSCs contain minor sub-populations that underwent WTp53-LOH in vitro. These cells may eventually give rise to malignant tumor cells in vivo. Indeed, prolonged in vitro cultivation of these clones revealed p53LOH.

Our in vivo results support the conclusion that the reprogramming process attenuates the loss of the WTp53 allele both in vitro and in vivo. In addition, it appears that in iPSCs p53LOH is sufficient to give rise to a malignant phenotype, although we cannot completely exclude the possibility that other driver mutations, occurring post p53LOH, may also contribute to malignancy.

MSCs are prone to undergo p53LOH. We next focused our study on ASCs, represented by HZp53-MSCs, which are known to be less genomically stable than ESCs and iPSCs. Moreover, familial sarcoma, which may arise from defective MSCs, ${ }^{15}$ is one of the diagnostic criteria of LFS. ${ }^{31}$ To that end, we established MSC isolates from adolescent and adult mice of various p53 genotypes (Supplementary Table 2).
Comparison of cell proliferation indicated that the WTp53-MSC isolates exhibited the slowest growth rate, whereas Mutp53-MSCs exhibited the most rapid. HZp53MSCs exhibited a bi-phasic growth pattern, with the first phase similar to WTp53 and the second similar to Mutp53 isolates, suggesting a switch in the p53 status of these cells (Figure 4a). Indeed, genotyping of individual HZp53 MSC isolates as a function of in vitro culturing time revealed that MSCs derived from adolescent mice lost the WTp53 allele at around passage 12, corresponding to the shift in the proliferation rates. MSCs isolated from adult mice lost WTp53 by passage 5, suggesting either a more rapid p53LOH process in MSCs originating from older mice or a higher fraction of cells, which underwent p53LOH before their isolation (Figures $4 \mathrm{~b}$ and $\mathrm{c}$ ).

Using antibodies that can distinguish between Mutp53 and WTp53 protein conformation, we found that, in agreement with the genotype profiling (Figure 4c), both proteins were detected at an early passage before $\mathrm{LOH}$ completion, whereas only the Mutp53 protein was present (Figure 4d) post LOH (passage 18).

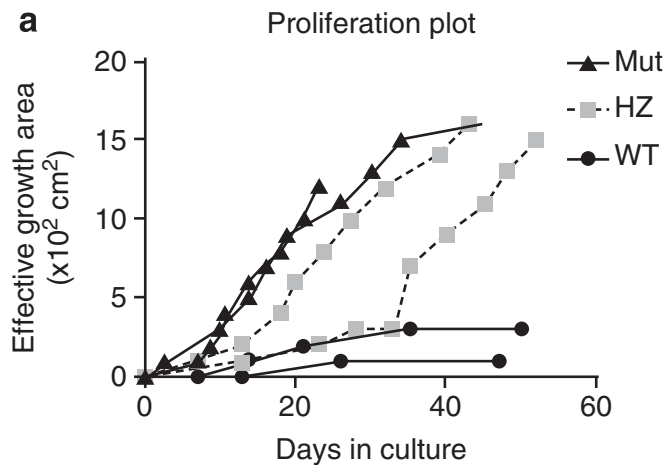

b $\quad \mathrm{LOH}$ of MSC isolates

C

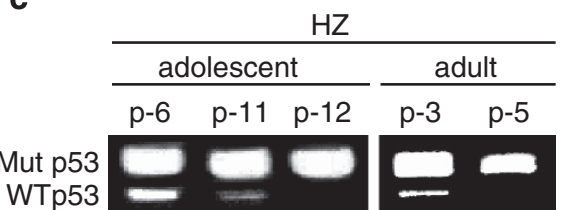

d

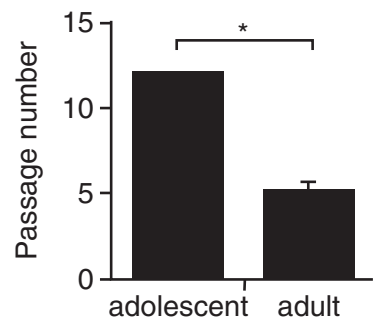

e $\%$ homozygous versus hemizygous p53 isolates post LOH

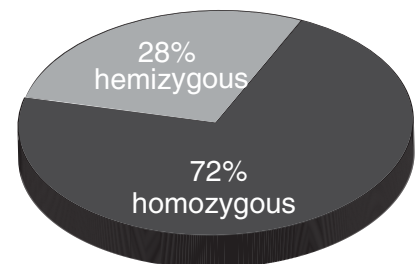

Figure 4 MSC isolates are prone to undergo p53LOH. MSC isolates derived from WTp53, HZp53 and Mutp53 adolescent (6-12 weeks) and adult (13-60 weeks) mice were established. Isolated clones were characterized for specific surface markers, to ensure they were free of contaminating hematopoietic and endothelial cells (Supplementary Figures 5 and 6 ). The MSC isolates were further characterized by their ability to differentiate into adipocytes and osteocytes (Supplementary Figures 7 and 8 ). (a) Proliferation plot presenting effective growth area over time in culture. Each curve represents MSC isolate derived from two mice. Two isolates were prepared for each mouse genotype. (b) p53 status of MSC isolates derived from two adolescent and four adult mice was determined by PCR genotyping every time the cells were passaged. The plot represents the mean passage number at which p53LOH completion was detected. (c) A representative figure of p53 PCR genotype analysis of MSC isolates derived from adolescent and adult mice. (d) Western blot of immune-precipitated (IP) p53 from adolescent HZp53 MSC isolate probed with Mutp53 (PAb-240), WTp53 (PAb-246) and lgG (control). (e) Percentage of homozygous and hemizygous HZp53 MSC isolates examined post LOH ( $n=7)$ using Taqman QRT-PCR as described in the legend of Figure 2d. ${ }^{*} P<0.05$ one-tail Student's $t$-test 
Characterization of the genetic mechanism that underlies p53LOH in MSCs revealed that $72 \%$ of examined clones duplicated the mutant (Mut) allele (Figure 4e). In two out of seven isolates, we observed a single Mutp53 copy after $\mathrm{LOH}$, while the Taqman probe recognizing exon 1 indicated that two copies were present. Thus, MSC isolates undergo WTp53$\mathrm{LOH}$ in an age-dependent manner, mainly through a duplication of the mutated allele (see Figures $2 e$ and $f$ ).

WTp53-LOH in HZp53-MSCs may lead to sarcomagenesis. Next, the tumorigenic potential of the various MSC isolates as a function of p53 status was examined. WTp53, HZp53 and Mutp53 MSC isolates derived from adolescent mice were subcutaneously injected into immune-compromised mice. All 10 mice injected with Mutp53-MSCs developed tumors (Figure 5A), whereas none of mice injected with WTp53-MSCs did (Figure 5A). HZp53 isolates were injected shortly after the completion of p53LOH. Despite the completion of the p53LOH process, no tumors were detected in mice injected with $\mathrm{HZp53} \mathrm{MSC}$ isolates derived from adolescent mice. In contrast, HZp53 MSC isolates derived from adult mice, which underwent WTp53-LOH 10 passages before injection, induced tumors (Figure $5 \mathrm{~A}$ ). These tumors exhibited histological characteristics of sarcoma with invasive edges, similar to tumors induced by Mutp53-MSCs (Figures 5B and C). Thus, the mere WTp53-LOH is not sufficient to promote tumorigenesis, but rather permits other tumor-promoting events to occur.

SKY analysis of the various HZp53 MSCs indicated a facilitated incidences of chromosome 11 translocation correlating with the age of mice the isolates were derived of (Supplementary Figure 6D). The highest number of chromosome 11 translocations was noticed in a tumor line derived from a MSC isolate obtained from aged mice (Supplementary Figures $3 \mathrm{C}$ and D). As indicated above, only HZp53-MSCs isolated from adult mice were tumorigenic (Figure 5a). Furthermore, MSCs from adult mice showed higher rate of chromosomal aberrations by SKY. Together these findings imply that $\mathrm{p} 53 \mathrm{LOH}$ permits other oncogenic events to occur, together leading to tumorigenic transformation at an advanced age.

\begin{abstract}
Alterations in the expression pattern of DNA repair genes coinciding with WTp53-LOH. To unravel the gene expression patterns associated with the $\mathrm{LOH}$ process, we performed mRNA profiling by CDNA microarray of MSC isolates derived from adolescent mice at passage 9, where the p53LOH process has already begun but was not yet completed (Figure 4c) and HZp53 isolate at passage 2, with no detectable LOH. Our analysis yielded 11 clusters. Cluster 1 , the 'downregulated cluster' (Figure 6a) was enriched for known 'p53 signaling' genes, such as Fas, Cong1, Cdkn1a,
\end{abstract}

A

\begin{tabular}{|c|c|c|c|}
\hline MSC & \multirow{2}{*}{$\begin{array}{c}\text { Mice age } \\
\text { strain }\end{array}$} & $\begin{array}{c}\text { Tumor } \\
\text { take }\end{array}$ & $\begin{array}{c}\text { Days to tumor } \\
\text { detection (mean) }\end{array}$ \\
\hline WT & & $0 / 10$ & ND \\
\hline HZ & \multirow{2}{*}{$8-10$} & $0 / 10$ & ND \\
\cline { 1 - 3 } Mut & & $10 / 10$ & $65-99(83)$ \\
\hline HZ & 59 & $10 / 10$ & $53-104(72.5)$ \\
\hline
\end{tabular}

B

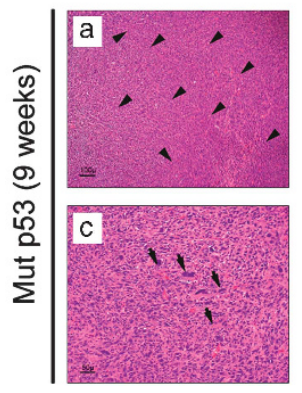

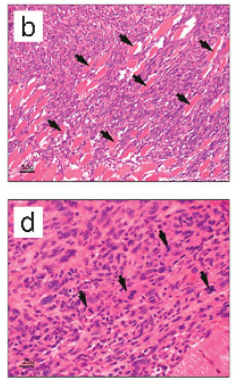

C

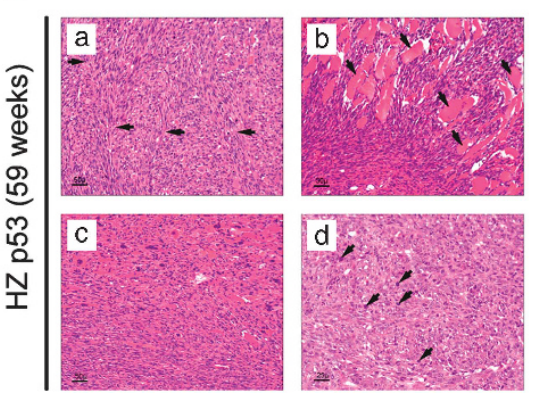

Figure 5 Aged adult mouse-derived HZp53 MSC isolates that underwent LOH form malignant sarcoma upon injection into immune-deficient mice. Cells of two MSC isolates each from WTp53, HZp53, Mutp53 adolescent mice and HZp53 adult mice were injected subcutaneously into NOD-SCID mice (5 per group, total of 10 mice for each p53 genotype) at passages 13-15. Tumors were removed and stained by H\&E. (A) Table summarizing the results of tumor take and days until detection of the tumors of the different MSC isolates. (B and C) Representative sections of tumors formed by Mutp53 (B) and HZp53 (C) MSC isolates. The cells injected are from the MSC isolates derived from 9-week-old Mutp53 mice and 59-week-old HZp53 mice. Tumors exhibited histological features typical of sarcoma. (a) Arrowheads indicate neoplastic cells arranged in interlacing fascicles, in an arrangement similar to fibrosarcoma. (b) A typical invasive edge is presented. The neoplastic cells engulfed pre-existing tissue elements; arrowheads indicate skeletal myofibers. (c) Marked difference in the overall size (anisocytosis) and nuclear size (anisokaryosis) of neoplastic cells. Several larger cells were identified (A, arrowheads). (d) Neoplastic cells exhibit a high mitotic rate (arrows indicate mitotic figures) 
a

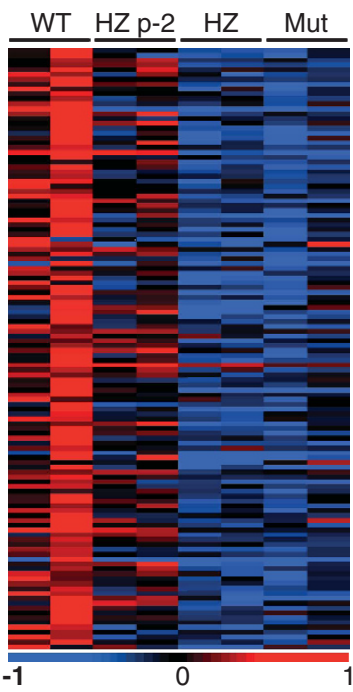

d Up regulated cluster

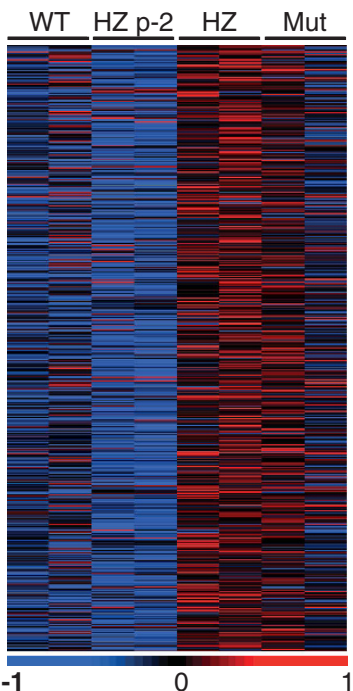

b
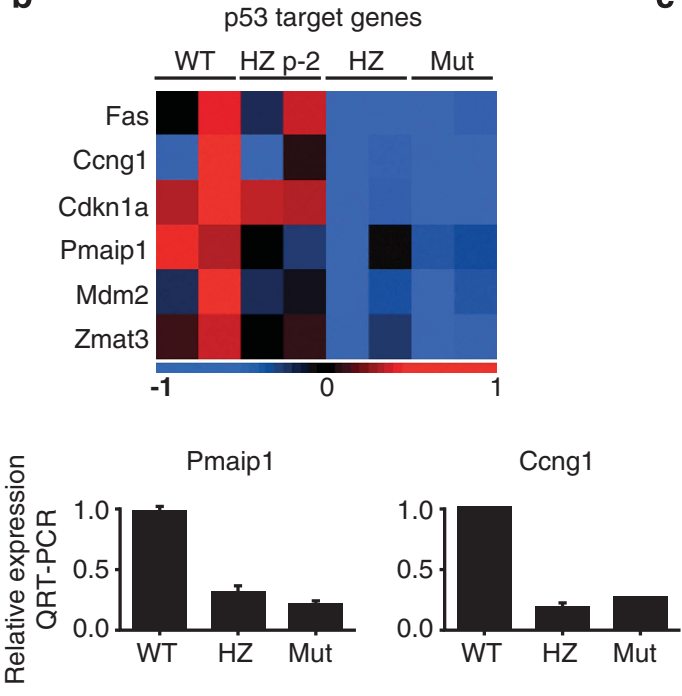
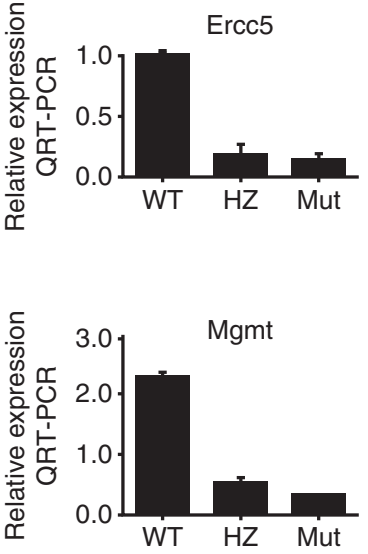

e

MSCs

up-regulated

cluster

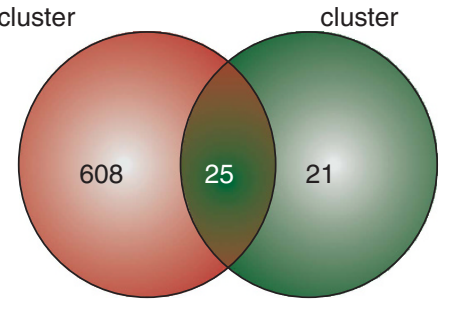

f

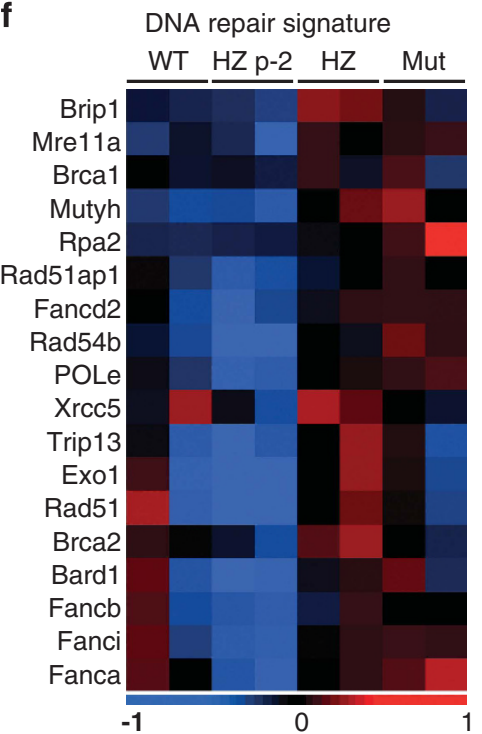

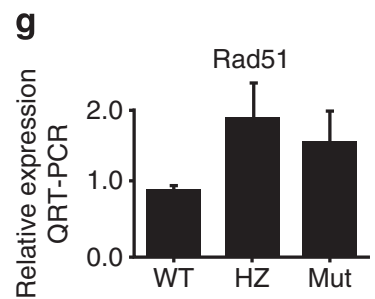
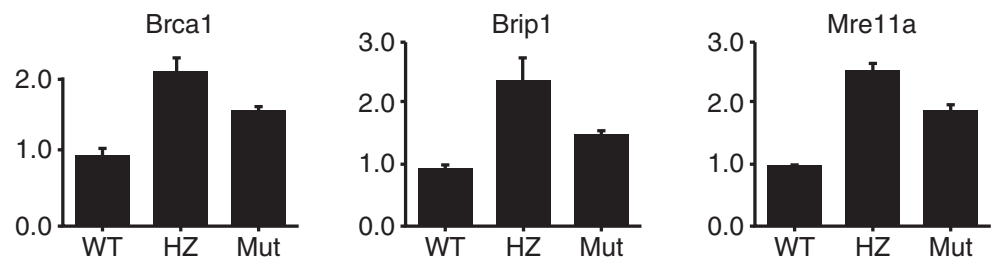

Figure 6 Downregulation of p53 target genes and upregulation of HR DNA repair genes coincide with p53LOH in MSC isolates. Genome-wide expression screen to identify changes associated with p53LOH. Samples originated from MSC isolates duplicates of WTp53 p-9, HZp53 p-2, HZp53 p-9 and Mutp53 p-9. The various samples were hybridized to the Agilent mouse Genome Array and the relative mRNA abundance of 55681 mRNA species was monitored. After standard preprocessing steps (see Materials and Methods), > 400000 expression values were collected from eight microarrays. Of these, 4524 genes exhibited a greater than twofold change between any two conditions, and were clustered according to their Pearson correlations. (a) Heat-map representation of Agilent microarray data depicting the downregulation of 133 genes between WTp53 (WT) and HZp53 p-2 (HZ p-2) to HZp53 p-9 (HZ) and Mutp53 (Mut) (cluster 1). (b) Heat-map representation of known p53 targets from cluster 1 and validation of gene expression of Pmaip1 and Ccng1 by QRT-PCR. (c) Validation of the expression changes in DNA repair genes from cluster 1 (Ercc5 and Mgmt) by QRT-PCR. (d) Upregulation of 633 genes between WTp53 (WT) and HZp53 p-2 (HZ p-2) to HZp53 p-9 (HZ) and Mutp53 (Mut) (cluster 2). (e) 'Upregulated cluster' was compared with the 'core proliferation cluster' (right hand). ${ }^{*} P<0.001$. A Fisher exact test was used to compare this overlap. (f) Heat-map representation of genes that were implicated as homologous recombination (HR) DNA repair pathway genes $\left(P=9.14 \times 10^{-4}\right)$ using IPA functional annotation, genes associated with non-homologous end joining (NHEJ), and genes associated with Fanconi Anemia crosslinked DNA repair known to engage HR. (g) QRT-PCR validation of expression changes in Rad51, Brca1, Brip1 and Mre11a. QRT-PCR results of each gene were normalized to Hprt. All samples were collected at p-9 unless indicated otherwise. Bars represent mean \pm S.D. 

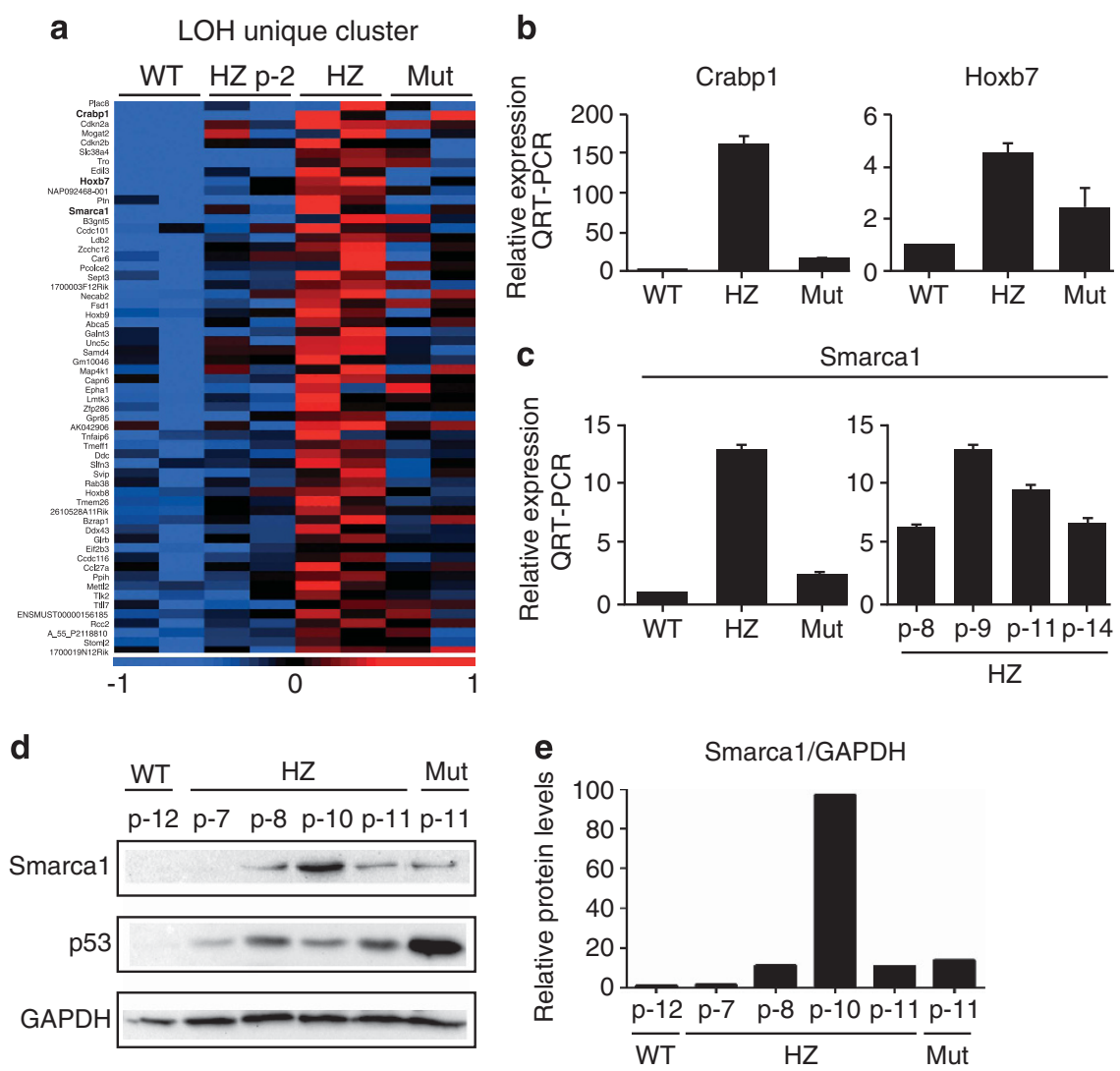

Figure 7 Elevated Smarca1 expression is associated with p53LOH in MSC isolates. A list of 'LOH unique' genes with highest expression in HZp53 p-9 was generated out of cluster 2. Only the genes with expression values at least 1.5-fold higher in HZp53 p-9 cells than in Mutp53 p-9 cells, and expression values at least 2-fold higher in HZp53 p-9 cells than in WTp53 p-9 and HZp53 p-2 cells, were selected. (a) A p53LOH-associated gene signature is presented as a heat-map of WTp53 p-9 (WT), HZp53 p-2 (HZ p-2), HZp53 p-9 (HZ) and Mutp53 p-9 (Mut) MSC isolates. (b and c) Validation of Crabp1, Hoxb7 and Smarca1 expression by QRT-PCR. The bars represent mean \pm s.d. (d) Western blot analysis of Smarca1 and p53. GAPDH was used as a loading control. (e) Quantities of Smarca1 protein levels were determined by measuring band intensity using the Lab Image 4.1 software

Pmaip1, Mdm2 and Zmat3 (Figure 6b), $P=2.76 \times 10^{-7}$ as well as for the p53-dependent DNA repair genes Ercc5 and Mgmt. Reduced expression of p53 target genes and the above DNA repair genes in HZp53 p-9 isolates confirm a progressive loss of functional WTp53 in these cells.

Cluster 2 represents a group of 633 upregulated genes (Figure 6d). We evaluated whether this 'upregulated cluster' contains genes previously described to be proliferationrelated genes ${ }^{32,33}$ and found an overlap of 25 genes (Figure 6e; Supplementary Table 3). Functional annotation revealed that the 'upregulated' cluster is enriched for genes of the HR DNA repair pathway (HRDRP; $P=9.14^{*} 10^{-04}$ ). Notably, the HRDRP genes do not overlap with the 'proliferation cluster' genes, with one exception (Trip13). We validated the elevated expression of Rad51, Brca1, Brip1 and Mre11a in p-9 HZp53-MSCs (Figure 6g). Fanconi Anemia proteins (Fanci, Fanca, Fancb and Fancd2) that execute cross-linked DNA repair, known to engage HR, ${ }^{34}$ also appeared in the upregulated cluster (Figure 6f) as well as $\operatorname{Xrcc5}$ (Ku80) and Mre11a, known to have a role in non-homologous end joining (NHEJ; Figure 6f). Figure $6 f$ illustrates a 'heat map' of expression levels of all the upregulated genes involved in HRDRP and additional DNA repair pathways, suggesting that HRDRP is one of the major mechanisms leading to $\mathrm{LOH}^{35}$
Expression of the chromatin remodeler Smarca1 is altered along the p53LOH process in MSC isolates.

As LOH involves chromosomal alterations, we also searched for genes controlling chromosomal integrity. A ' $\mathrm{LOH}$ unique' list, with the highest expression level in HZp53 p-9 was derived of the 'upregulated' cluster (Figure 7a). This identified the chromatin remodeler Smarca1 ${ }^{36}$ We found that the expression of Smarca1 mRNA and protein peaked at passages $8-9$, followed by a decline after p53LOH was attained in the majority of the population (Figures 7c-e). No change in the expression patterns of other major chromatin remodelers, ${ }^{36}$ such as Smarca2, Smarca4 and Smarca5, was observed (Supplementary Figure 9). This indicates a unique correlation between Smarca1 expression and p53LOH.

Bi-directional p53LOH. As both iPSCs and MSC isolates represent in vitro SC models, it was important to examine whether p53LOH may occur in vivo. BM progenitors were isolated and tested ex vivo for their p53 status. As we observed that p53LOH in MSCs is age dependent, we evaluated the in vivo $\mathrm{p} 53 \mathrm{LOH}$ process as a function of age in mice.

We could not detect any p53LOH in colony-forming units-fibroblast (CFU-Fs) derived from adolescent HZp53 
mice. However, CFU-Fs derived from BM of adult HZp53 mice exhibited p53LOH at varying frequencies (Figures $8 \mathrm{a}$ and $\mathrm{c}$ ). Surprisingly, the majority of p53LOH (average of $6.2 \%$ versus $1.7 \%$ ) events led to the loss of the Mutp53 allele (Figures $8 b-d)$. This suggests that a specific mechanism dictates a preference to retain the WT allele, assuring genome integrity in vivo.

Surprisingly, genomic analysis of 156 iPSC single-cell sub-clones of an iPSC clone that retained its heterozygosity indicated that the majority kept their HZp53 genotype, however three of them have lost their Mut allele (Figure 8e). In order to ensure that it is not the loss of only the LoxP gene sequences, masquerading as the loss of the Mutp53 allele, we sequenced the area around the $\mathrm{R} 172 \mathrm{H}$ mutation
(Supplementary Figure 10) and found solely WTp53 DNA sequences. Similarly, genotyping of 220 single-cell subclones of early passage MSC isolates also identified the loss of the Mutp53 allele (1.4\%; Figure 8f). All together our results suggest that bi-directional $\mathrm{LOH}$ is not restricted to a specific type of SC.

In sum, we observed p53LOH in somatic cells, iPSCs and MSCs. However, the p53LOH frequency was different between the systems and correlates with their genome stability. The highest frequency was observed in somatic cells; it was lower in ASC, in an age-dependent manner and lowest in embryonic-like iPSCs. Only cells that underwent p53LOH gave rise to malignant tumors. In most cases, p53LOH resulted of HRDRP. Interestingly, in BM progenitors

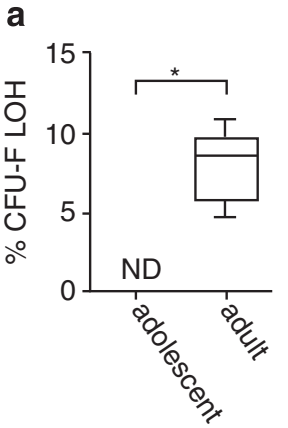

b

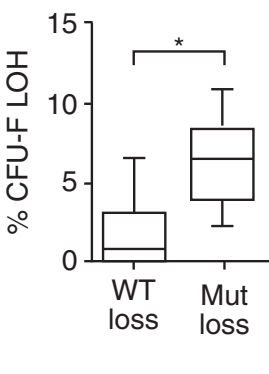

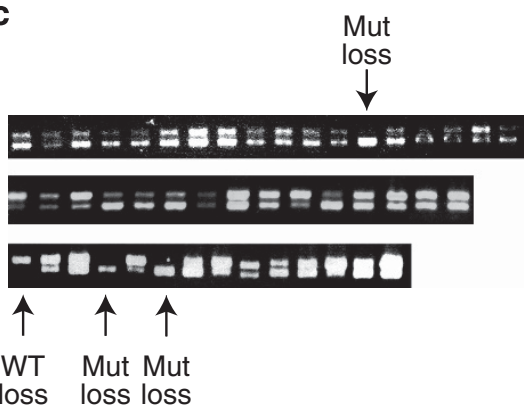

Mouse no. 9

\begin{tabular}{|c|c|c|c|c|c|}
\hline $\begin{array}{c}\text { Mouse } \\
\text { number }\end{array}$ & Mice age & $\begin{array}{c}\text { Number of } \\
\text { screened } \\
\text { CFU-F }\end{array}$ & $\begin{array}{c}\text { Total } \\
\text { LOH } \\
(\%)\end{array}$ & $\begin{array}{c}\text { Loss of } \\
\text { WT allele } \\
(\%)\end{array}$ & $\begin{array}{c}\text { Loss of } \\
\text { mutant } \\
\text { allele (\%) }\end{array}$ \\
\hline 1 & Adolescent & 74 & 0 & 0 & 0 \\
2 & (5-12 weeks) & 39 & 0 & 0 & 0 \\
3 & 82 & 0 & 0 & 0 \\
4 & & 94 & 6.4 & 0 & 0 \\
\hline 5 & & 120 & 9.1 & 1.7 & 7.5 \\
6 & Adult & 46 & 10.8 & 0 & 10.8 \\
7 & (13-60 weeks) & 88 & 4.5 & 0 & 4.5 \\
8 & & 47 & 8.4 & 2.1 & 6.3 \\
9 & & 45 & 8.8 & 6.6 & 2.2 \\
10 & & \multicolumn{4}{|r}{} \\
\hline
\end{tabular}

e

LOH of iPSC single-cell sub-clones

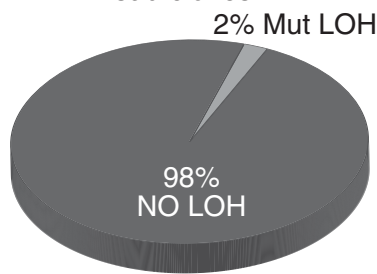

\section{f}

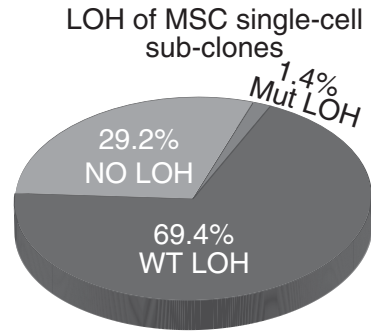

Figure 8 WTp53 and Mutp53-LOH in BM mesenchymal progenitors, iPSCs and MSCs. BM was isolated from HZp53 adolescent and adult mice and CFU-F formation was assessed. Two weeks later, colonies were genotyped by PCR. (a) Percentage of BM-derived CFU-F colonies that underwent p53LOH, derived from adolescent and adult mice. (b) Percentage of CFU-F colonies derived from BM of adult mice that lost their either WTp53 or Mutp53 allele. Box plots represent median, 25th percentile, 75th percentile and extreme values. (c) Raw unprocessed data of PCR-genotyped CFU-F colonies derived from mouse no. 9. WT or Mut LOH is marked accordingly. (d) Table summarizing the results obtained from genotyped CFU-Fs presented in panels b and c. (e) Two HZp53-iPSC clones were single-cell sub-cloned in 96 well-plates. After 2-3 weeks, plates were genotyped by PCR. A summary of 156 single-cell sub-clones is presented in a pie chart. (f) Two HZp53 MSC isolates derived from adolescent mice were sub-cloned at a density of either one or five cells per well in 96-well plates, and PCR-genotyped. Summary of the data from three independent experiments of 220 single-cell sub-clones is presented in a pie chart. The diagram summarizes the percentage of sub-clones that did not undergo LOH (NO LOH), sub-clones that lost the WTp53 (WT LOH) and sub-clones that lost the Mutp53 (Mut LOH). ND, not detected. ${ }^{*} P<0.05$ one-tail Student's $t$-test 
of adult mice, p53LOH was surprisingly frequent. Strikingly, the loss of the Mutp53 allele was four times more frequent than the loss of the WT allele in these cells. Our findings highlight that bi-directional p53LOH can determine whether a cell lose its predisposition to cancer or initiate events leading to malignant transformation.

\section{Discussion}

Although LFS patients seem to mature normally, they develop a wide spectrum of cancer types in childhood and adult life where about $60 \%$ tumors demonstrate $\mathrm{p} 53 \mathrm{LOH} .{ }^{8}$ Sarcomas, which are of mesenchymal origin, are prevalent tumors in LFS patients and murine models. ${ }^{6-8,37}$ The possibility of a link between cancer development and deregulation of SCs ${ }^{11-13}$ challenged us to study the significance of p53LOH in SCs and tumorigenesis. The availability of the reprogramming process that enables to recapitulate a transition from adult somatic cells into embryonic-like cells and HZp53 mice allowed us to address the above question, both in vitro and in vivo, as a function of age. Indeed, we observed that although p53LOH is attenuated with the onset of reprogramming, the in vitro p53LOH process is active in MSCs. Moreover, p53LOH was more pronounced in MSCs established from BM of adult mice compared with adolescent mice most likely reflecting a higher incidence of p53LOH in vivo. Furthermore, our data suggest a link between p53LOH, aging and tumorigenesis. It was previously reported that transformation of MSCs seemed to be highly dependent on alterations in the p53/p21 pathway; mainly through inactivation of WTp53 ${ }^{14}$ and that MSCs might require few genetic alterations to undergo transformation. ${ }^{14}$ Moreover, others have shown that only after long-term in vitro cultivation, p53 knockout MSCs were able to form tumors in mice. $^{38}$ We observed that HZp53-MSCs obtained from adolescent mice, injected shortly after the completion of p53LOH, did not give rise to tumors. This agrees with the notion that MSCs require several genetic alterations for transformation that can be acquired gradually after p53 restrain is released. Indeed, injection of HZp53-MSCs isolates obtained from older mice, injected at the same passage as all other isolates but having undergone $\mathrm{p} 53 \mathrm{LOH} 10$ passages earlier, gave rise to aggressive tumors similar to those induced by Mutp53-MSCs. Interestingly, a specific reduction in p53 levels in MSCs but not in the spleen of old mice was reported. ${ }^{39}$ These results suggest that loss of WTp53 in MSCs is an initiating step in sarcomagenesis.

The link between p53LOH, tumorigenesis and aging observed in our in vitro SC system led us to examine this link in vivo. We examined BM progenitors from $\mathrm{HZp} 53$ mice at various ages and demonstrated, for the first time, that p53LOH occur in vivo. This process is accelerated with age, reaching up to $10 \%$ of the progenitor SCs in adult mice. Studies in yeast have revealed an increase in $\mathrm{LOH}$ as the mother cell ages. ${ }^{40}$ Surprisingly, the majority of cells that underwent p53LOH lost the Mut allele. This phenomenon of Mutp53-LOH was also observed in single-cell sub-clones of our in vitro SC systems. Although this is the first report on the loss of the Mutp53 allele through LOH in an apparent healthy tissue, Mut-LOH was noticed for other TSGs with a role in DNA repair. ${ }^{41-45}$ It is tempting to speculate that $\mathrm{LOH}$ can be seen as a physiological genetic repair mechanism. Gene expression, copy number and sequencing analyses (Figures $6 f, 2 e, f$ and $h$ ) point to the induction of HRDRP events as the mechanism underlie most cases of $\mathrm{LOH}$. Mutp53 seems to induce a state of chronic DNA insults, as cells harboring either Mutp53 or HZp53 exhibit a DNA repair gene expression signature. The observed gene-signature is manifested by the upregulation of specific genes involved in the dsDNA breakresponse, which includes both $\mathrm{HR}$ and NHEJ. Although $\mathrm{HR}$ is a high-fidelity DNA repair mechanism, NHEJ is highly error prone, and thus these two mechanisms may have significantly different consequences. Studies in the Drosophila male germline have indicated that HR increases linearly with age. The authors speculated that in young individuals, selective pressures may favor usage of NHEJ and single-strand annealing, which are faster but more error prone. However, in old individuals, the fidelity obtained by HR is favorable. ${ }^{46}$ Recently, a link between HR, reprogramming and p53 was established, wherein cells defective in their HR pathway yield a smaller number of reprogrammed cells. This decrease is mediated by p53-dependent growth arrest and apoptosis, which is responsible for the elimination of cells with damaged DNA. In the absence of p53, the reprogramming process continues at the expense of accumulating genetic aberrations. $^{47}$

The accessibility of DNA repair enzymes to damaged DNA is blocked by nucleosomes, thus chromatin remodeling must occur during the detection and repair of damaged DNA. ${ }^{48,49}$ Indeed, we found that the 'unique p53LOH' list contains the chromatin remodeler Smarca1, which belongs to the ISWI ATP remodeling family. ${ }^{36}$

In summary, we present for the first time the evidence for a physiological bi-directional p53LOH process, which may serve as a cell fate checkpoint in SCs. At large, p53HZ-SCs, although carrying a Mutp53 allele, manage to suppress its activity and exhibit a normal phenotype. However, with age, cells become less stable and may activate $\mathrm{LOH}$ as a second line of defense in an attempt to lose the mutated allele. $\mathrm{LOH}$ can be regarded as an event that helps maintain genomic stability. Indeed, this LOH DNA repair mechanism was restricted to less genomically stable cells. For example, BM progenitors of adolescent mice, which are genomically more stable than BM of adult mice, did not undergo p53LOH. Similarly, reprogramming of HZp53-MEFs, which robustly undergo p53LOH, mostly restrained the p53LOH process. However, in cases where the WT allele is lost, cells acquire properties leading to tumor formation. Our observation that $\mathrm{LOH}$ is attenuated in embryonic iPSCs while adult MSCs readily undergo $\mathrm{LOH}$, coupled with the fact that p53LOH yields cells lacking WTp53, can explain why LFS patients do not acquire tumors during development, yet would develop tumors (sarcomas in particular) later in life.

\section{Materials and Methods}

Mice strains. The following mice strains were used in this study: C57BL/6 containing WTp53, HZp53 or Mutp53 alleles (kindly provided by Professor G Lozano) Hfh11nu Nude mice and NOD.CB17-prkdc-SCID/NCrHsd (Harlan, Rehovot, Israel). Animal protocols were approved by the Institutional Animal Care and Use Committee of the Weizmann Institute of Science. 
Cell cultures. MEFs prepared as previously described ${ }^{25}$ and were maintained in DMEM (Biological Industries, Bet-Haemek, Israel) supplemented with 10\% FCS and antibiotics.

MSCs were grown in MSC medium, containing murine MesenCult Basal Media (StemCell Technologies, Vancouver, BC, Canada) supplemented with 20\% murine mesenchymal supplement (StemCell Technologies), $60 \mu \mathrm{g} / \mathrm{ml}$ penicillin, $100 \mu \mathrm{g} / \mathrm{ml}$ streptomycin and $50 \mu \mathrm{g} / \mathrm{ml}$ kanamycin. Cells were incubated at $37^{\circ} \mathrm{C}$ in a humidified atmosphere of $10 \% \mathrm{CO}_{2}$. Fresh medium was added twice a week. iPSCs were maintained on irradiated MEFs in ES medium: DMEM (Biological Industries) containing 15\% FCS, $5 \mathrm{mg}$ recombinant human LIF (Millipore, Bellerica, MA, USA LIF1005), $1 \mathrm{mM}$ glutamine (Biological Industries), 1\% nonessential amino acids (Biological Industries), $0.1 \mathrm{mM} \beta$-mercaptoethanol (Invitrogen), $60 \mu \mathrm{g} / \mathrm{ml}$ penicillin and $100 \mu \mathrm{g} / \mathrm{ml}$ streptomycin (Biological Industries).

Generation and characterization of iPSCs. The EF1a-STEMCCA lentiviral vector, a kind gift of Dr. Mostoslavsky $G{ }^{50}$ allows for constitutive expression of the four proteins Oct4, Klf4, Sox2 and mCherry from a single polycistronic transcript. Lentiviruses were produced in 293T packaging cells as previously described. ${ }^{51}$ Forty-eight hours post infection, $3.5 \times 10^{5}$ cells were plated in $10 \mathrm{~cm}$ plates, on top of a feeder layer of irradiated MEFs (irradiated with 60 gray gamma irradiation), and medium was replaced to ES medium. At this stage, the medium was supplemented with 2l: small-molecule inhibitors CHIR99021 (GSK3binhibitor, $3 \mathrm{mM}$; Axon Medchem, Groningen, Netherlands) and PD0325901 (ERK1/2 inhibitor, $1 \mathrm{mM}$; TOCRIS Bristol, UK). Emerging colonies were selected by morphology. Each colony was isolated either mechanically or by incubating for $15 \mathrm{~min}$ in a trypsin filled glass cylinder on the emerging clone. The colonies were then transferred to 12-well plates containing $\mathrm{ES}+21$ medium and separately passaged. A few passages later, 21 was removed gradually from the medium. Alkaline phosphatase activity was performed as previously described. ${ }^{52}$ Colony number was determine using Image-Pro Plus analysis software (Media Cybernetics, Rockville, MD, USA). Further characterization and verification of the nature of the reprogrammed clones were performed by QRT-PCR as described below in detail.

Preparation of BM cells suspension. BM nucleated cells were obtained from femurs and tibias of 6-8 weeks old, 4-5 months old and 13 months old WTp53, HZp53 and Mutp53 mice. The bone was flushed with PBS containing $2 \%$ FCS (Biological Industries LTD). The cells were dissociated to single-cell suspension and were centrifuged at $300 \mathrm{~g}$ for $5 \mathrm{~min}$ at room temperature. Red blood cells (RBCs) were removed by RBC lysis buffer (R7757, Sigma, St. Louis, MO, USA).

Production and characterization of MSC isolates. For the standard MSC production, the pellet BM cells were re-suspended to single-cell suspension and seeded in six-well plates containing MSC medium (ratio of one mice to one well). The medium was replaced every 3 days to remove the non-adherent cells. Once the adherent cells had reached confluence, the cells were trypsinized using Trypsin B solution ( $0.05 \%$ EDTA, $0.25 \%$ trypsin), centrifuged for 5 min at $300 \times g$, $4{ }^{\circ} \mathrm{C}$, re-suspended in their medium and split $1: 2$. Expression of MSC isolates surface markers was analyzed using the following antibodies: anti-CD11b-PE, antiCD45.2-PE, anti-CD31-PE, anti-CD34-PE, anti-Ter119-PE, anti-Sca1-PE, Rat IgG2b isotype control-PE, Rat IgG2a isotype control-PE and Mouse IgG2a isotype control (eBioscience, San Diego, CA, USA). $10^{6}$ MSC cells were harvested and incubated for $10 \mathrm{~min}$ on ice with the Fc blocker antibody of anti-CD16/CD32, following $1 \mathrm{~h}$ incubation on ice with the specific antibodies listed above. Cells were subjected to flow cytometry analysis using a LSRII flow cytometer (BD, Franklin Lakes, NJ, USA, Immunocytometry Systems).

\section{Evaluation of MSC differentiation potential}

Adipogenesis: Cells were seeded at a concentration of $2 \times 10^{4} \mathrm{cells} / \mathrm{well}$ in a 24-well plate. The next day, adipogenic medium containing $10 \mu \mathrm{g} / \mathrm{ml}$ insulin (Sigma), $0.5 \mathrm{mM}$ IBMX (Sigma) and $1 \times 10^{-5} \mathrm{M}$ dexamethasone (Sigma) was added. The cells were grown for 1-3 weeks, with medium replacement twice a week. Adipogenesis was detected by Oil red 0 staining. For Oil red 0 quantification, 4\% IGEPAL CA 630 (Sigma) in isopropanol was added to each well. Light absorbance was measured in $492 \mathrm{~nm}$.

Osteogenesis: Cells were seeded at a concentration of $2 \times 10^{4} \mathrm{cells} / \mathrm{well}$ in a 24-well plate. The next day, osteogenic medium containing $50 \mu \mathrm{g} / \mathrm{ml} \mathrm{L}$-ascorbic acid-2 phosphate, $10 \mathrm{mM}$ glycerol 2-phosphate disodium salt and $1 \times 10^{-7} \mathrm{M}$ dexamethasone (all from Sigma) was added. The cells were grown for 1-3 weeks with medium being replaced twice a week. Osteogenic differentiation was detected by Alizarin red staining. For Alizarin red quantification, $0.5 \mathrm{~N}$ hydrochloric acid $(\mathrm{HCl})$ and $5 \%$ SDS were added to each well. Light absorbance was measured in $405 \mathrm{~nm}$.

Population doubling time and growth area measurement. Proliferation rates of the various MEFs were evaluated by calculating population doubling time. Cells $\left(5 \times 10^{5}\right)$ were plated in $6 \mathrm{~cm}$ plates in duplicates. The cells were counted every 3 or 4 days and re-plated at the same density. This procedure was repeated five times. MSCs proliferation rates were evaluated by defining the number of times that the cells were transferred to a larger growth area at defined time points. For instance, if the growth area doubled itself then the effective growth area increased by one unit of $6 \mathrm{~cm}$ plate $\left(28.3 \mathrm{~cm}^{2}\right)$.

Single-cell cloning: IPSC and MSC isolates were serially diluted to reach 1-5 cells per well. The cells were plated on gelatin-coated 96 -well plates. The colonies were examined by microscope to ensure that they originated form a single cell. Two to three weeks later, colonies were subjected to genomic DNA genotyping. CFU-Fs assay. BM nucleated cells of HZp53 adolescent (four mice at the age of 5-12 weeks old) and adult (six mice at the age of 13-60 weeks old) were plated at cell densities of $20 \times 10^{6}-30 \times 10^{6}$ in $10 \mathrm{~cm}$ BD falcon plates (BD). The cells were grown in MSC medium as described above and re-fed once a week without further treatment. At day 14, un-fixed colonies were subjected to genomic DNA genotyping.

In vivo tumorigenesis assays: iPSC clones were trypsinized and re-plated with ES medium for $15 \mathrm{~min}$. The non-adherent cells were collected, resuspended in PBS and injected sub-coutaneously into 6-8 weeks old Hfh11nu Nude mice $\left(10^{6} \mathrm{cells} / 100 \mu \mathrm{l}\right.$, with Matrigel matrix at a ratio of $1: 1$; Becton Dickenson FAL354232). The tumors were removed 2-16 weeks post injection, fixed in 4\% paraformaldehyde, decalcified and embedded in paraffin. Selected sections, derived from three distinct tumor levels were stained with hematoxylin and eosin (H\&E).

Seven- to eight-week-old age NOD.CB17-prkdc-SCID/NCrHsd mice were injected subcutaneously with $3 \times 10^{6}$ MSC cells expressing WTp53, HZp53 or Mutp53. Mice were killed when their tumors reached a diameter of $10 \mathrm{~mm}$ or after 120 days after inoculation. Upon tumor removal, half the tumor was mechanically disaggregated on mesh to establish MSC-transformed cell lines. The remaining portion of the tumor was used for histological analysis by H\&E staining.

Western blot and immunoprecipitation analysis: Cells were lysed in $1 \times$ passive lysis buffer (Promega, Madison, WI, USA), $1 \mathrm{mM} \mathrm{Tris-HCl}(\mathrm{pH} \mathrm{7.5)}$, $5 \mathrm{Mm} \mathrm{MgCl}_{2}, 0.13 \mathrm{mM} \mathrm{CaCl}_{2}, 25 \mu \mathrm{g} / \mu$ l DNase (Sigma), incubated for $1 \mathrm{~h}$ at $37^{\circ} \mathrm{C}$ and $0.5 \times$ TLB buffer was added $(50 \mathrm{mmol} / \mathrm{T} \mathrm{Tris}-\mathrm{HCl}, 100 \mathrm{mmol} / \mathrm{I} \mathrm{NaCl}, 1 \%$ Triton X-100, $0.5 \%$ sodium deoxycholate, $0.1 \%$ SDS) supplemented with protease inhibitor cocktail (Sigma) and phosphatase inhibitor cocktails I and II (Sigma) for 15 min on ice, followed by centrifugation. BCA reagent (Pierce, Rockford, IL, USA) was used to determine Protein concentration. Fifty micrograms protein of each sample were separated by SDS-gel electrophoresis, and transferred to nitrocellulose membranes. The following primary antibodies were used: anti-mouse p53 monoclonal 1c12 (Cell Signaling Technology, Danver, MA, USA), anti-Smarca1 polyclonal (Biorbyt, Cambridge, UK) and anti-GAPDH mab374 (ChemiconTemecula, CA, USA). The protein-antibody complexes were detected using horseradish peroxidase-conjugated secondary antibodies and the Amersham ECL western blotting detection reagents (GE Healthcare, Wauwatosa, WI, USA). For immunoprecipitation, PAb240, a monoclonal anti-Mutp53 antibody (a kind gift from Dr. D Lane), PAb246, a monoclonal anti-WTp53 antibody (a kind gift from Dr. D Lane) or control IgG antibody (Sigma) were incubated overnight at $4^{\circ} \mathrm{C}$ with the lysate followed by the addition of $30 \mu$ protein A beads for $2 \mathrm{~h}$ at $4{ }^{\circ} \mathrm{C}$. The immunoprecipitated material was washed and pellets were resuspended in SDS sample buffer and subjected to western blot analysis.

Genomic DNA extraction: Cells were resuspended in lysis buffer $(100 \mathrm{mM}$ Tris-HCl, pH 7.5, $100 \mathrm{mM}$ EDTA, $100 \mathrm{mM} \mathrm{NaCl}, 0.5 \%$ SDS, Sigma) and incubated at $65^{\circ} \mathrm{C}$ for $30 \mathrm{~min}$, following by incubation in $4{ }^{\circ} \mathrm{C}$ for $15 \mathrm{~min}$ with $\mathrm{LiCl} / \mathrm{KAC}$ solution (Sigma). DNA was precipitated with isopropanol and washed with $70 \%$ ethanol. The genomic DNA was re-suspended in DDW.

Genomic sequencing. Prior sequencing, a PCR analysis was performed using $5 \mathrm{ng}$ genomic DNA and primers surrounding the p53 R172H mutation site. Forward primer: $5^{\prime}$-TCCCAGTCCTCTCTTTGCTG-3'. Reverse primer: 5 '-CTCGGGTGGCTCATAAGGTA-3'. PCR reactions consisted of 30 cycles of $95^{\circ} \mathrm{C}$ for $30 \mathrm{~s}, 60^{\circ} \mathrm{C}$ for $30 \mathrm{~s}$ and $72^{\circ} \mathrm{C}$ for $90 \mathrm{~s}$, purified with HiYield 
gel/PCR DNA fragment extraction kit (RBCBioscience, Xindian, Taiwan). Twenty nanograms of DNA was sequenced at the sequencing unit of Weizmann Institute of Science with either forward or reverse primer above.

Quantifying copy numbers in genomic DNA using the TaqMan copy number assay. Copy number genotyping was performed using RT-PCR-based copy number analysis (TaqMan Copy Number Assays, Applied Biosystems, Foster City, CA, USA) for p53 (exon 1- intron 1 Chr.11:69394017, Applied Biosystems) and custom loxP site Mut p53 (intron 4, Applied Biosystems). Analyses were initially performed on a subset of mouse tip fibroblasts of known genotypic origin. For each single-well reaction using $20 \mathrm{ng}$ genomic DNA and $1 \times$ TaqMan Universal PCR Master Mix, a $1 \times$ TaqMan Copy Number Assay, which contained forward primer, reverse primer and FAM dye-labeled MGB probe specific for the gene of interest, was run simultaneously with a $1 \times$ TaqMan Copy Number Reference Assay, which contained forward primer, reverse primer and a VIC dye-labeled TAMRA probe specific for transferrin receptor (TFRC) 17 according to the manufacturer's instructions. PCR was performed in 96-well plates using a PCR system (7300 Real-Time PCR System, Applied Biosystems). Samples were assayed using triplicate wells for each gene of interest. Copy numbers were estimated (CopyCaller Software version 2.0, Applied Biosystems) using the $\Delta \mathrm{Ct}$ relative quantification method. A maximum likelihood algorithm was used to estimate the mean $\Delta \mathrm{Ct}$ expected for copy number $1(\mathrm{CN}=1)$ based on the probability density distribution across all samples, and this parameter was used in subsequent copy number calculations for each given gene. This analytical method was used to calculate the relative copy number of a target gene normalized to TFRC, a reference of known copy number $(\mathrm{CN}=2)$.

Genomic DNA genotyping: Genotyping was performed by PCR analysis using $50 \mathrm{ng}$ of genomic DNA and primers surrounding the loxP site (see Supplementary Figure 1). Forward primer: $5^{\prime}$-ACCTGTAGCTCCAGCAC TGG-3'. Reverse primer: 5'-ACAAGCCGAGTAACGATCAGG-3'. PCR reactions consisted of 35 cycles of $95^{\circ} \mathrm{C}$ for $60 \mathrm{~s}, 60^{\circ} \mathrm{C}$ for $60 \mathrm{~s}$ and $72{ }^{\circ} \mathrm{C}$ for $180 \mathrm{~s}$ and run on $2 \%$ agarose electrophoresis gel.

Reverse transcription and QRT-PCR: Total RNA was isolated using the Nucleospin II kit (Macherey Nagel, Duren, Germany) according to the manufacturer's protocol. An aliquot of $2 \mu \mathrm{g}$ of total RNA was reverse-transcribed using Bio-RT (BioLab, Jerusalem, Israel) and random hexamer plus oligodTprimers (NEB, Ipswich, MA, USA). QRT-PCR was performed using SYBR Green PCR Master Mix (Applied Biosystems) on an ABI 7300 instrument (Applied Biosystems). The values for the specific genes were normalized to HPRT housekeeping gene control. Specific primers were designed for the following genes: Pmaip1: forward: $5^{\prime}$-GCAGAGCTACCACCTGAGTTC-3', reverse: $5^{\prime}$-CTTT TGCGACTTCCCAGGCA-3', Cong1: forward: 5'-ACAACTGACTCTCAGAAACT GC-3', reverse: 5'-CATTATCATGGGCCGACTCAAT-3', Ercc5: forward: $5^{\prime}$-TG CTGGCCGTGGATATTAGC-3', reverse: $5^{\prime}$-GCCGGTGGAATAATGTGAGAAGA-3', Mgmt: forward: 5'-TGCTCTCCATCACCCTGTGTT-3', reverse: 5'-AACACCTGT CTGGTGAATGAATCTT-3', Rad51: forward: 5'-AAGTTTGGTCCACAGCCTA TTT-3', reverse: $5^{\prime}$-CGGTGCATAAGCAACAGCC-3' ${ }^{\prime}$, Brca1: forward: $5^{\prime}$-CGAATCT GAGTCCCCTAAAGAGC-3', reverse: 5'-AAGCAACTTGACCTTGGGGTA-3', Brip1: forward: $5^{\prime}$-TACTCTGGCTGCAAAGTATCTG-3', reverse: $5^{\prime}$-TCGTGCATCTACA TGGTGGAC-3', Mre11a: forward: $5^{\prime}$-CCTCTTATCCGACTACGGGTG-3', reverse: 5'-ACTGCTTTACGAGGTCTTCTACT-3', Crabp1: forward: 5'-CAGCAGCGAGAATT TCGACGA-3', reverse: $5^{\prime}$-CGCACAGTAGTGGATGTCTTGA-3', Hoxb7: forward: 5'-AAGTTCGGTTTTCGCTCCAGG-3', reverse: 5'-ACACCCCGGAGAGGTTCTG-3', Smarca1: forward: 5'-TGCTACAAATGATCCGTCATGG-3', reverse: $5^{\prime}$-GCGTTCT CGTTTAGGAGGTTCA-3', Smarca2: forward: 5'-AGCCAGATGAGTGACCTGC-3', reverse: $5^{\prime}$-TGCTTGGCATCCTTTTCGGAA-3', smarca4: forward: $5^{\prime}$-CAAAGACA AGCATATCCTAGCCA-3' ${ }^{\prime}$, reverse: $5^{\prime}$-CACGTAGTGTGTGTTAAGGACC-3', smarca5: forward: $5^{\prime}$-GACACCGAGATGGAGGAAGTA-3', reverse: 5'-CGAACAGCTCTGT CTGCTTTA-3'.

Flow cytometric determination of apoptosis by annexin V/propidium iodide double staining. Cells were analyzed for phosphatidylserine exposure by an Annexin-V FITC/propidium iodide Annexin using V FLUOS staining kit (Roche, Basel, Switzerland) according to the manufacturer's protocol.

DNA Library Preparation and Sequencing: Exome capturing was carried out with Agilent SureSelect V4 All Exon Mus musculus kit (Agilent, Santa Clara, CA, USA) according to the manufacturer's protocol. In brief, 2-5 $\mu \mathrm{g}$ of gDNA were fragmented to $\sim 170 \mathrm{bp}(\mathrm{PE})$ insert-size with a Covaris S2 device (Covaris, Woburn, MA, USA). $500 \mathrm{ng}$ of Illumina adapter-containing libraries (Illumina, San Diego, CA, USA) were hybridized with the exome baits at $65^{\circ} \mathrm{C}$ for $24 \mathrm{~h}$.
Each enriched final paired-end library was sequenced using multiplexing of six samples on two Illumina Hiseq2000 lanes (Illumina).

Mapping and analysis: Illumina sequence data were aligned to the $\mathrm{mm} 10$ mouse reference genome assembly using BWA $\left(0.5 .9,{ }^{53}\right)$ duplicate and nonuniquely mapping reads were excluded. We subsequently detected SNVs and InDels as described in references Rausch et al..$^{54}$ and Jones et al. ${ }^{55}$ adjusting the pipeline by using mouse genome annotations for Mapability, simple tandem repeats, repeat masker, segmental duplications, dbSNP137 as well as mm10 SNVs by ENSEMBL.

cDNA microarray. Total RNA was extracted using Tri-Reagent (MRC Global Inc., Houston, TX, USA) according to the manufacturer's protocol, and submitted for analysis to the Micro-Array unit of Weizmann Institute of Science, Rehovot, Israel. Agilent chips were used as a platform for RNA loading. The limma package ${ }^{56}$ was used for microarray processing. Background was subtracted using the function backgroundCorrect and normalization within and between arrays was performed using the functions normalizeWithinArrays and normalizeBetweenArrays, respectively. Spots with the same probes were averaged. Analysis of variance including contrasts was applied to the data set using Partek Genomic Suite 6.5 (Partek Inc., St. Charles, MO, USA).

SKY analysis: Half a million cells were plated in a $10-\mathrm{cm}$ plate and were cultivated for $48 \mathrm{~h}$ following replacement of medium and additional incubation period of $24 \mathrm{~h}$. Colcemid $(0.1 \mu \mathrm{g} / \mathrm{ml})$ was added to the culture for $4 \mathrm{~h}$. Cells were trypsinized and lysed with hypotonic buffer following fixation in glacial acetic acid/methanol $(1: 4)$. The chromosomes were simultaneously hybridized with 24 combinatorially labeled chromosome painting probes and analyzed using the SD200 spectral bioimaging system (Applied Spectral Imaging Ltd, Carlsbad, CA, USA).

Statistical analysis. Unless specified otherwise, all statistical analyses were performed using Graphpad Prism Software Inc (La Jolla, CA, USA).

\section{Conflict of Interest}

The authors declare no conflict of interest.

Acknowledgements. We express our gratitude to D Zippori for his will to collaborate and help. We thank G Lozano for her generosity in making the Mutp53 mice available. We are grateful to $O$ Brenner for his pathological analysis of the tumors, to G Friedlander for bioinformatics analysis, to I Bar-am for SKY analysis, to I Ino for his help in animal experiments, to El Nahle for every waking hour. This study was supported by a Center of Excellence of the Flight-Attendant Medical Research Institute (FAMRI), and by the Israel Science Foundation ISF-MOKED center from the Israeli Academy of Science. Y Shetzer is supported by the German-Israeli Helmholtz Research School in Cancer Biology. V Rotter is the incumbent of the Norman and Helen Asher Professorial Chair for Cancer Research at the Weizmann Institute.

1. Hanahan D, Weinberg RA. Hallmarks of cancer: the next generation. Cell 2011; 144 646-674.

2. Berger AH, Knudson AG, Pandolfi PP. A continuum model for tumour suppression. Nature 2011; 476: 163-169.

3. Berger AH, Pandolfi PP. Haplo-insufficiency: a driving force in cancer. J Pathol 2011; 223: 137-146.

4. Tuna M, Knuutila S, Mills GB. Uniparental disomy in cancer. Trends Mol Med 2009; 15: $120-128$.

5. Malkin D, Li FP, Strong LC, Fraumeni JF Jr, Nelson CE, Kim DH et al. Germ line p53 mutations in a familial syndrome of breast cancer, sarcomas, and other neoplasms. Science 1990; 250: 1233-1238.

6. Lang GA, Iwakuma T, Suh YA, Liu G, Rao VA, Parant JM et al. Gain of function of a p53 hot spot mutation in a mouse model of Li-Fraumeni syndrome. Cell 2004; 119: $861-872$.

7. Olive KP, Tuveson DA, Ruhe ZC, Yin B, Willis NA, Bronson RT et al. Mutant p53 gain of function in two mouse models of Li-Fraumeni syndrome. Cell 2004; 119: 847-860.

8. Varley JM, Evans DG, Birch JM. Li-Fraumeni syndrome-a molecular and clinical review. $\mathrm{Br}$ J Cancer 1997; 76: 1-14.

9. Cervantes RB, Stringer JR, Shao C, Tischfield JA, Stambrook PJ. Embryonic stem cells and somatic cells differ in mutation frequency and type. Proc Natl Acad Sci USA 2002; 99: 3586-3590. 
10. Giachino C, Orlando L, Turinetto V. Maintenance of genomic stability in mouse embryonic stem cells: relevance in aging and disease. Int J Mol Sci 2013; 14: 2617-2636.

11. Reya T, Morrison SJ, Clarke MF, Weissman IL. Stem cells, cancer, and cancer stem cells. Nature 2001; 414: 105-111.

12. Som A, Wen S, Tu SM. Stem cell origin of testicular seminoma. Clin Genitourin Cance 2013; 11: 489-494.

13. Stange DE, Clevers $\mathrm{H}$. The Yin and Yang of intestinal (cancer) stem cells and their progenitors. Stem Cells 2013; 31: 2287-2295.

14. Rodriguez R, Rubio R, Masip M, Catalina $P$, Nieto A, de la Cueva $T$ et al. Loss of p53 induces tumorigenesis in p21-deficient mesenchymal stem cells. Neoplasia 2009; 11: 397-407.

15. Rodriguez R, Rubio R, Menendez $P$. Modeling sarcomagenesis using multipotent mesenchymal stem cells. Cell Res 2012; 22: 62-77.

16. Rubio R, Garcia-Castro J, Gutierrez-Aranda I, Paramio J, Santos M, Catalina P et al. Deficiency in $\mathrm{p} 53$ but not retinoblastoma induces the transformation of mesenchymal stem cells in vitro and initiates leiomyosarcoma in vivo. Cancer Res 2010; 70: 4185-4194.

17. Rubio R, Gutierrez-Aranda I, Saez-Castillo Al, Labarga A, Rosu-Myles M, Gonzalez-Garcia $S$ et al. The differentiation stage of $\mathrm{p} 53-\mathrm{Rb}$-deficient bone marrow mesenchymal stem cells imposes the phenotype of in vivo sarcoma development. Oncogene 2012; 32: 4970-4980.

18. Li H, Fan X, Kovi RC, Jo Y, Moquin B, Konz R et al. Spontaneous expression of embryonic factors and $\mathrm{p} 53$ point mutations in aged mesenchymal stem cells: A model of age-related tumorigenesis in mice. Cancer Res 2007; 67: 10889-10898.

19. Takahashi K, Yamanaka S. Induction of pluripotent stem cells from mouse embryonic and adult fibroblast cultures by defined factors. Cell 2006; 126: 663-676.

20. Wernig $\mathrm{M}$, Meissner $\mathrm{A}$, Foreman $\mathrm{R}$, Brambrink $\mathrm{T}$, Ku M, Hochedlinger $\mathrm{K}$ et al. In vitro reprogramming of fibroblasts into a pluripotent ES-cell-like state. Nature 2007; 448 318-324.

21. Mayshar Y, Ben-David U, Lavon N, Biancotti JC, Yakir B, Clark AT et al. Identification and classification of chromosomal aberrations in human induced pluripotent stem cells. Cell Stem Cell 2010; 7: 521-531.

22. Laurent LC, Ulitsky I, Slavin I, Tran H, Schork A, Morey R et al. Dynamic changes in the copy number of pluripotency and cell proliferation genes in human ESCs and iPSCs during reprogramming and time in culture. Cell Stem Cell 2011; 8: 106-118.

23. Hussein SM, Batada NN, Vuoristo S, Ching RW, Autio R, Narva E et al. Copy numbe variation and selection during reprogramming to pluripotency. Nature 2011; 471: 58-62.

24. Gore A, Li Z, Fung HL, Young JE, Agarwal S, Antosiewicz-Bourget J et al. Somatic coding mutations in human induced pluripotent stem cells. Nature 2011; 471: 63-67.

25. Sarig R, Rivlin N, Brosh R, Bornstein C, Kamer I, Ezra O et al. Mutant p53 facilitates somatic cell reprogramming and augments the malignant potential of reprogrammed cells. J Exp Med 2010; 207: 2127-2140.

26. Krizhanovsky V, Lowe SW. Stem cells: The promises and perils of p53. Nature 2009; 460 1085-1086.

27. Hanna J, Saha K, Pando B, van Zon J, Lengner CJ, Creyghton MP et al. Direct cell reprogramming is a stochastic process amenable to acceleration. Nature 2009; 462 595-601.

28. Brosh R, Assia-Alroy Y, Molchadsky A, Bornstein C, Dekel E, Madar S et al. p53 Counteracts reprogramming by inhibiting mesenchymal-to-epithelial transition. Cell Death Differ 2012; 20: 312-320.

29. Liyanage M, Coleman A, du Manoir S, Veldman T, McCormack S, Dickson RB et al. Multicolour spectral karyotyping of mouse chromosomes. Nat Genet 1996; 14: 312-315.

30. Rotter V, Wolf D, Pravtcheva D, Ruddle FH. Chromosomal assignment of the murine gene encoding the transformation-related protein p53. Mol Cell Biol 1984; 4: 383-385.

31. Schneider K, Zelley K, Nichols KE, Garber J. Li-Fraumeni Syndrome. In: Pagon RA Adam MP, Bird TD, Dolan CR, Fong CT, Stephens K (eds) GeneReviews. Seattle (WA), University of Washington, 1993.

32. Whitfield ML, George LK, Grant GD, Perou CM. Common markers of proliferation. Nat Rev Cancer 2006; 6: 99-106.

33. Brosh R, Rotter V. Transcriptional control of the proliferation cluster by the tumo suppressor p53. Mol bioSystems 2010; 6: 17-29.
34. Yang YG, Herceg Z, Nakanishi K, Demuth I, Piccoli C, Michelon J et al. The Fanconi anemia group A protein modulates homologous repair of DNA double-strand breaks in mammalian cells. Carcinogenesis 2005; 26: 1731-1740.

35. Tischfield JA. Loss of heterozygosity or: how I learned to stop worrying and love mitotic recombination. Am J Hum Genet 1997; 61: 995-999.

36. Okabe I, Bailey LC, Attree O, Srinivasan S, Perkel JM, Laurent BC et al. Cloning of human and bovine homologs of SNF2/SWI2: a global activator of transcription in yeast $\mathrm{S}$. cerevisiae. Nucleic Acids Res 1992; 20: 4649-4655.

37. van Boxtel R, Kuiper RV, Toonen PW, van Heesch S, Hermsen R, de Bruin A et al. Homozygous and heterozygous p53 knockout rats develop metastasizing sarcomas with high frequency. Am J Pathol 2011; 179: 1616-1622.

38. Armesilla-Diaz A, Elvira G, Silva A. p53 regulates the proliferation, differentiation and spontaneous transformation of mesenchymal stem cells. Exp Cell Res 2009; 315: 3598-3610.

39. Wilson A, Shehadeh LA, Yu H, Webster KA. Age-related molecular genetic changes of murine bone marrow mesenchymal stem cells. BMC Genomics 2010; 11: 229.

40. McMurray MA, Gottschling DE. An age-induced switch to a hyper-recombinational state. Science 2003; 301: 1908-1911.

41. Yan $H$, Jin H, Xue G, Mei Q, Ding F, Hao L et al. Germline hMSH2 promoter mutation in a Chinese HNPCC kindred: evidence for dual role of LOH. Clin Genet 2007; 72: 556-561.

42. Sanchez de Abajo A, de la Hoya M, van Puijenbroek M, Godino J, Diaz-Rubio E, Morreau $\mathrm{H}$ et al. Dual role of $\mathrm{LOH}$ at MMR loci in hereditary non-polyposis colorectal cancer? Oncogene 2006; 25: 2124-2130.

43. Boettger MB, Sergi $C$, Meyer P. BRCA1/2 mutation screening and LOH analysis of lung adenocarcinoma tissue in a multiple-cancer patient with a strong family history of breast cancer. J Carcinog 2003; 2: 5.

44. Clarke CL, Sandle J, Jones AA, Sofronis A, Patani NR, Lakhani SR. Mapping loss of heterozygosity in normal human breast cells from BRCA1/2 carriers. Br J Cancer 2006; 95: 515-519.

45. Loveday C, Turnbull C, Ruark E, Xicola RM, Ramsay E, Hughes D et al. Germline RAD51C mutations confer susceptibility to ovarian cancer. Nat Genet 2012; 44: 475-476; author reply 476 .

46. Preston CR, Flores $C$, Engels WR. Age-dependent usage of double-strand-break repair pathways. Curr Biol 2006; 16: 2009-2015.

47. Gonzalez F, Georgieva D, Vanoli F, Shi ZD, Stadtfeld M, Ludwig T et al. Homologous recombination DNA repair genes play a critical role in reprogramming to a pluripotent state. Cell Rep 2013; 3: 651-660.

48. Erdel F, Rippe K. Binding kinetics of human ISWI chromatin-remodelers to DNA repair sites elucidate their target location mechanism. Nucleus 2011; 2: 105-112.

49. Toiber D, Erdel F, Bouazoune K, Silberman DM, Zhong L, Mulligan P et al. SIRT6 Recruits SNF2H to DNA Break Sites, Preventing Genomic Instability through Chromatin Remodeling. Mol Cell 2013; 51: 454-468.

50. Sommer CA, Stadtfeld M, Murphy GJ, Hochedlinger K, Kotton DN, Mostoslavsky G. Induced pluripotent stem cell generation using a single lentiviral stem cell cassette. Stem Cells 2009; 27: 543-549.

51. Mostoslavsky G, Fabian AJ, Rooney S, Alt FW, Mulligan RC. Complete correction of murine Artemis immunodeficiency by lentiviral vector-mediated gene transfer. Proc Nat Acad Sci USA 2006; 103: 16406-16411.

52. Kochupurakkal BS, Sarig R, Fuchs O, Piestun D, Rechavi G, Givol D. Nanog inhibits the switch of myogenic cells towards the osteogenic lineage. Biochem Biophys Res Commun 2008; 365: 846-850.

53. Li H, Durbin R. Fast and accurate short read alignment with Burrows-Wheeler transform. Bioinformatics 2009; 25: 1754-1760.

54. Rausch T, Jones DT, Zapatka M, Stutz AM, Zichner T, Weischenfeldt J et al. Genome sequencing of pediatric medulloblastoma links catastrophic DNA rearrangements with TP53 mutations. Cell 2012; 148: 59-71.

55. Jones DT, Hutter B, Jager N, Korshunov A, Kool M, Warnatz HJ et al. Recurrent somatic alterations of FGFR1 and NTRK2 in pilocytic astrocytoma. Nat Genet 2013; 45: 927-932.

56. Smyth GK, Speed T. Normalization of cDNA microarray data. Methods 2003; 31: 265-273.

Supplementary Information accompanies this paper on Cell Death and Differentiation website (http://www.nature.com/cdd) 